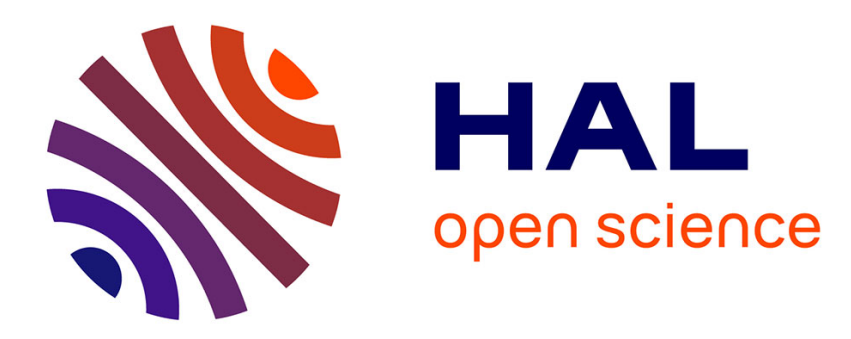

\title{
Hyperinflation argentine de 1989 : une interprétation post-keynésienne \\ Jonathan Marie
}

\section{To cite this version:}

Jonathan Marie. Hyperinflation argentine de 1989 : une interprétation post-keynésienne. Revue de la régulation. Capitalisme, institutions, pouvoirs, 2014, 15 (1er semestre), 10.4000/regulation.10794. hal-01346920

\section{HAL Id: hal-01346920 https://sorbonne-paris-nord.hal.science/hal-01346920}

Submitted on 8 Apr 2021

HAL is a multi-disciplinary open access archive for the deposit and dissemination of scientific research documents, whether they are published or not. The documents may come from teaching and research institutions in France or abroad, or from public or private research centers.
L'archive ouverte pluridisciplinaire HAL, est destinée au dépôt et à la diffusion de documents scientifiques de niveau recherche, publiés ou non, émanant des établissements d'enseignement et de recherche français ou étrangers, des laboratoires publics ou privés. 


\section{Hyperinflation argentine de 1989 : une interprétation post-keynésienne}

Argentina's hyperinflation of 1989: a Post-Keynesian interpretation

Hiperinflación argentina de 1989 : una interpretación post-keynesiana

\section{Jonathan Marie}

\section{(2) OpenEdition}

Journals

Édition électronique

URL : http://journals.openedition.org/regulation/10794

DOI : 10.4000/regulation. 10794

ISSN : 1957-7796

Éditeur

Association Recherche \& Régulation

Référence électronique

Jonathan Marie, "Hyperinflation argentine de 1989 : une interprétation post-keynésienne », Revue de la régulation [En ligne], 15 | 1er semestre / Spring 2014, mis en ligne le 17 juillet 2014, consulté le 23 avril 2020. URL : http://journals.openedition.org/regulation/10794 ; DOI : https://doi.org/10.4000/ regulation.10794

Ce document a été généré automatiquement le 23 avril 2020

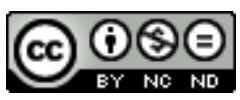

Revue de la régulation est mise à disposition selon les termes de la Licence Creative Commons Attribution - Pas d'Utilisation Commerciale - Pas de Modification 4.0 International. 


\title{
Hyperinflation argentine de 1989 : une interprétation post- keynésienne
}

\author{
Argentina's hyperinflation of 1989: a Post-Keynesian interpretation \\ Hiperinflación argentina de 1989 : una interpretación post-keynesiana
}

\author{
Jonathan Marie
}

\section{NOTE DE L'AUTEUR}

Une version préliminaire de cet article a été discutée à l'occasion de la $6^{\mathrm{e}}$ conférence internationale du CEMF organisée à l'université de Bourgogne en décembre 2012.

Je remercie tout particulièrement A. Asensio, S. Charles et J.-F. Ponsot pour leurs remarques et suggestions effectuées alors. Je remercie aussi M. Houssiaux ainsi que les deux rapporteurs anonymes pour leurs lectures attentives. Bien sûr, je demeure seul responsable des insuffisances de cet article.

\section{Introduction}

1 L'article propose d'appréhender l'hyperinflation argentine de 1989 par l'étude de ses origines historiques depuis les années 1970. Il cherche plus précisément à poursuivre deux objectifs.

D'abord un objectif théorique: présenter une vision post-keynésienne de l'hyperinflation. L'article s'attache à montrer que l'hyperinflation présente une nature différente de celle d'un phénomène inflationniste, même violent. L'hyperinflation, c'est l'expression du rejet d'une monnaie au profit d'un substitut. Pour saisir ce processus, l'article souligne que l'hyperinflation est rendue possible par la conjonction de trois éléments. Premier élément, l'existence d'un processus inflationniste virulent. Selon la 
théorie post-keynésienne, l'inflation est provoquée par le conflit de répartition (Rowthorn, 1977 ; Dutt, 1992), conflit qui porte sur la répartition de la valeur ajoutée entre travailleurs et capitalistes. Deuxième élément, s'ajoutent à l'inflation générée par le conflit de répartition des phénomènes d'indexation des prix et des salaires. Ils tendent à entretenir mais aussi à renforcer perpétuellement l'inflation, comme l'ont souligné les auteurs néo-structuralistes (notamment Frenkel, 1979; Carvalho, 1993). Cependant, si l'inflation se renforce ainsi, on ne comprend pas ce qui peut faire dégénérer le processus provoquant le rejet complet de la monnaie domestique au profit d'une devise (si l'indexation est efficace, la répartition du revenu est en quelque sorte gelée et les prix peuvent perpétuellement continuer à être exprimés en unités de monnaie domestique). Pour comprendre ce mécanisme, il faut prendre en compte l'importance du change, suivant ainsi Robinson (1966). Dans une économie fortement inflationniste, un cercle vicieux inflation - dégradation du change réel - augmentation de l'endettement externe en devises peut se mettre en place. Alors, les anticipations sur le change peuvent devenir autoréalisatrices et déclencher le run sur les réserves. Ce sont donc ces anticipations sur le change qui forment le troisième élément constitutif de l'hyperinflation.

3 Nous défendons dans cet article la proposition théorique suivante ${ }^{1}: l^{\prime}$ hyperinflation se développe au sein d'une économie endettée en devises et en situation de déficit du solde courant de la balance des paiements, dans laquelle on observe un conflit de répartition virulent auquel s'ajoutent des phénomènes d'indexation et d'anticipations sur le change.

4 Le second objectif de cet article est de confronter cette approche théorique du phénomène hyperinflationniste à l'épisode observé en Argentine en 1989. L'étude d'économie politique historique permet de montrer que le conflit de répartition a été particulièrement virulent, au moins depuis la fin des années 1970 jusqu'en 1989. Cependant, l'inflation n'a pas été toujours croissante sur cette période. L'analyse va rappeler qu'on a pu temporairement la diminuer grâce à la mise en place de différentes politiques économiques. L'une d'elle, jouant directement sur le conflit de répartition, a cependant favorisé l'apparition de nouvelles contraintes (endettement externe accru, implantation du dollar comme support et unité de réserve de valeur notamment). Ce fut le cas avec la politique de libéralisation externe adoptée simultanément à un ancrage du change particulier (politique dite de la Tablita en vigueur de 1978 à 1981).

5 Au milieu des années 1980, l'inflation sera très nettement réduite grâce à un plan de réforme économique dit "hétérodoxe». Ce plan, adopté en 1985, supprime les éléments d'indexation en œuvre dans l'économie et promet la stabilisation du change. Mais nous observerons que si les phénomènes d'indexation peuvent être effacés (au moins à court terme), une issue du conflit de répartition faiblement inflationniste n'est pas pour autant obtenue dans un contexte où les anticipations sur le change sont dorénavant largement développées (le dollar est l'actif de réserve de valeur par excellence). Dès lors, lorsque le taux de change ne peut être soutenu par la Banque centrale, l'inflation se renforce instantanément. L'étude de la trajectoire économique argentine permet de soutenir notre argument: l'hyperinflation naît de la présence simultanée d'un conflit de répartition violent, de phénomènes d'indexation importants et de l'impossibilité à maintenir un taux de change stable de la monnaie domestique par rapport à une devise clef. 
6 Le plan de l'article est le suivant: la première section présente notre approche théorique. La deuxième est consacrée à l'analyse de la trajectoire macroéconomique argentine à partir du milieu des années 1970, qui aboutit à l'hyperinflation de 1989. La dernière section regroupe les remarques conclusives.

\section{Comprendre l'émergence de l'hyperinflation : une proposition cohérente avec la théorie post- keynésienne}

7 L'hyperinflation est selon le critère traditionnel et orthodoxe de Cagan (1956) une très violente inflation, observée lorsque le taux d'inflation mensuel dépasse $50 \%$. En accord avec la théorie quantitative de la monnaie, l'hyperinflation, comme l'inflation, puisque sa nature est la même, est provoquée par une politique budgétaire laxiste et/ou une politique monétaire lâche.

8 Ces arguments ne sont pas compatibles avec la théorie post-keynésienne. D'une part parce que celle-ci considère l'offre de monnaie endogène. Dans ce cadre, un excès d'offre de monnaie ne peut apparaître, et donc encore moins être responsable d'un phénomène inflationniste ou hyperinflationniste. D'autre part, comme nous le rappelons par la suite, l'inflation est au sein de la théorie post-keynésienne la conséquence du conflit de répartition; elle n'est donc pas d'origine monétaire. Là non plus, il n'y a pas lieu de croire que ce pourrait être le cas pour l'hyperinflation.

9 Si les auteurs post-keynésiens ont peu axé leurs recherches sur la compréhension des phénomènes hyperinflationnistes ${ }^{2}$ en économie ouverte, Joan Robinson a toutefois établi de solides fondations pour développer une construction théorique permettant de comprendre l'émergence de tels phénomènes. En 1938, dans un commentaire publié dans l'Economic Journal concernant l'ouvrage de Bresciani-Turroni (1937) consacré à l'hyperinflation allemande ${ }^{3}$, elle propose une interprétation alternative à l'analyse orthodoxe des sources de l'hyperinflation. Selon Robinson, celle-ci se développe sur un terreau particulier : aux origines, il y a la présence d'un conflit de répartition virulent qui provoque une inflation importante. Une séquence particulière est alors susceptible d'émerger: l'inflation va provoquer une détérioration du taux de change réel, qui induit une dégradation du solde courant de la balance des paiements. Sous la pression, le change nominal ne peut être tenu, et une dépréciation (ou une dévaluation si l'on est en change fixe) doit suivre afin de contrecarrer la dégradation du solde courant. Seulement, cet ajustement peut alimenter la spirale inflationniste, car il provoque une augmentation des prix des biens importés. Si les biens importés sont des inputs utilisés par les firmes domestiques, celles-ci vont chercher à augmenter les prix afin de conserver leurs marges. Si les biens importés sont des biens de consommation finale, les travailleurs vont chercher à obtenir des hausses de salaires afin de conserver leur pouvoir d'achat, augmentations qui vont provoquer une hausse des coûts des firmes déclenchant une hausse des prix.

10 La réalisation d'un tel cercle vicieux est identifiée par Robinson (1966, p. 74) lors de l'hyperinflation allemande: "Each rise in wages, therefore, precipitated a further fall in the exchange rate, and each fall in the exchange rate called forth a further rise in wages. "

11 Dans cet article, nous souhaitons adopter mais aussi développer cette approche théorique, puis tenter d'observer si celle-ci fournit un éclairage intéressant concernant 
l'hyperinflation survenue en Argentine en 1989. Pour cela, nous rappelons d'abord ce qu'est le conflit de répartition et comment celui-ci est susceptible de générer un processus inflationniste.

\section{1. Le conflit de répartition responsable de l'inflation}

$12 \mathrm{Au}$ sein de la théorie post-keynésienne, l'inflation reflète l'existence du conflit de répartition et est générée dans un cadre institutionnel et historique donné. Le conflit de répartition porte sur le partage de la valeur ajoutée et oppose les capitalistes, rémunérés grâce à la propriété du capital, aux travailleurs, rémunérés grâce à l'utilisation par les firmes du travail qu'ils offrent. L'analyse des conflits de répartition est, dès la fin des années 1950, un élément d'identification d'un courant post-keynésien se référant aux écrits de Keynes mais aussi à ceux de Kalecki.

13 Les auteurs post-keynésiens ont cherché à développer des modèles explicatifs de l'inflation dont le modèle de référence a été proposé par Rowthorn (1977). Le conflit de répartition oppose donc capitalistes et travailleurs. Si l'un de ces groupes parvient à augmenter le taux d'inflation sans que l'autre ne l'ait anticipé, n'ait pu l'anticiper ou n'ait pu agir pour s'en prémunir, cela provoquera une redistribution du revenu réel en sa faveur. Le tout est de savoir comment on parvient à agir sur le taux d'inflation. Casetti (2003) estime que le modèle de Rowthorn s'inscrit clairement dans une vision kaleckienne de l'économie, soulignant que l'approche retenue combine théorie de la demande effective et approche conflictuelle de l'inflation.

Le niveau général des prix est déterminé par les coûts, eux-mêmes supportés par les capitalistes. Le principal déterminant de ces coûts est le coût salarial. À ce coût, les firmes appliquent un taux de marge afin de déterminer les prix (Weintraub, 1978). Une variation des prix intervient si le taux de marge varie, si la productivité du travail change ou si le salaire nominal évolue. C'est ainsi qu'intervient le conflit de répartition dans le processus inflationniste : chacun tente d'obtenir une répartition du revenu qui lui soit plus favorable. Les salariés en obtenant des salaires plus élevés, les capitalistes en augmentant leurs profits, via des augmentations de prix. La capacité d'un groupe à atteindre son objectif dépend de son pouvoir de négociation («bargaining power »). Les salariés peuvent bénéficier d'un pouvoir de négociation élevé, entre autres exemples, si le chômage dans l'économie considérée est faible (Rowthorn, 1977), si le chômage diminue (Cassetti, 2003), en fonction du type d'organisations syndicales (Susjan et Lah, 1997) ou en fonction du cadre institutionnel (Setterfield, 2007). Le pouvoir de marché des capitalistes est quant à lui affecté positivement par l'existence de marchés oligopolistiques ou par le taux d'utilisation du capital (si on se place dans une situation de sous-utilisation du capital, les firmes tendent à augmenter la production plutôt que les prix suite à une augmentation de la demande).

15 Dans le modèle fondateur de Rowthorn, les travailleurs négocient un salaire nominal pour la période suivante. Puis les firmes fixent les prix, cherchant à atteindre le niveau de prix compatible avec le taux de marge désirét. Le taux d'inflation est alors la conséquence d'une divergence entre les objectifs des entreprises (le taux de marge visé) et des travailleurs (le salaire réel recherché). L'« Aspiration Gap » est la mesure de cette divergence.

16 Dutt (1992) ou Lavoie (1992) proposent un modèle qui synthétise les principaux apports post-keynésiens concernant l'inflation. Relevons que le conflit de répartition est 
toujours actuellement au cœur de la réflexion théorique menée par les auteurs postkeynésiens. À titre d'exemple, Cassetti (2012) adopte clairement la même conception du conflit de répartition que celle retenue ici. Celui-ci est d'ailleurs un enjeu fondamental dans l'analyse et la catégorisation des régimes de croissance (profit-led ou wage-led). L'article de Cassetti souligne la difficulté d'obtenir un compromis sur le partage de la valeur ajoutée compatible avec le plein-emploi. Actuellement, les régimes de croissance sont plutôt profit-led et peu inflationnistes, ce qui, pour ce dernier point, n'était pas nécessairement le cas par le passé, comme nous le montrerons pour l'Argentine. Mais le cadre théorique permettant d'analyser ces différentes situations peut bien sûr être conservé.

17 Les firmes fixent donc les prix. Elles les augmentent lorsque le taux de marge observé est inférieur au taux de marge désiré. Et comme le souligne Dalziel (1990, p. 425), une cible de taux de marge peut être analysée comme une cible de taux de salaire réel. En d'autres termes, afin d'augmenter le taux de marge, les firmes doivent parvenir à diminuer les salaires réels. De plus, le pouvoir de marché est associé à la capacité d'établir un prix ; dès lors, c'est cette capacité qui détermine ou au moins influence le salaire réel. Pour résumer, le taux d'inflation dépend d'une part et positivement du pouvoir de marché des firmes sur les marchés des biens, et d'autre part, de l'écart entre le salaire effectif réel et le salaire réel désiré par les firmes (ou le taux de marge recherché par les firmes). Cela signifie que plus le salaire réel sera supérieur à la cible de salaire réel des entreprises et plus le taux d'inflation sera élevé, ou que plus le taux de marge visé par les entreprises sera fort (i.e. plus le niveau de salaire réel visé sera faible), plus le taux d'inflation sera, là encore, élevé.

Soulignons toutefois que si la rémunération du travail est un élément important des coûts des entreprises, il en existe bien sûr d'autres. Et si ces coûts varient, cela va aussi affecter les marges réalisées. Les entrepreneurs peuvent donc chercher à transférer une augmentation de leurs coûts non salariaux sur la rémunération réelle des travailleurs. L'impact d'une variation des taux d'intérêt sur le processus de négociation des salaires et des prix a notamment été souligné par Galbraith (1957) ou Dutt (1990-1991). Si les taux d'intérêt s'élèvent, les firmes vont chercher à répercuter cette hausse de leurs coûts financiers via une hausse des prix. Le raisonnement est bien sûr valable si on considère l'évolution des prix des inputs des firmes ou encore l'évolution du taux de change de la monnaie domestique. Ainsi, l'exchange rate pass-through est expliqué de la manière suivante dans une perspective post-keynésienne (voir par exemple Arestis et Milberg, 1994): la dépréciation de la monnaie domestique va affecter la rentabilité des firmes car les inputs importés (ou les biens d'investissement), dont les prix sont libellés en devises, ont un prix exprimé en monnaie domestique qui s'élève. Les firmes vont alors chercher à contrecarrer cette tendance en "passant » une partie de la diminution du taux de marge sur les prix. Vera (2010, p.604) parvient lui aussi au même résultat (une dépréciation externe de la monnaie provoque de l'inflation), mais cette fois en montrant que celle-ci peut être déclenchée par la baisse du salaire effectif réel. Sa modélisation souligne comment une situation d'état stationnaire peut être affectée par une dépréciation externe de la monnaie.

19 Retenons enfin que travailleurs comme firmes ne disposent pas d'un pouvoir de négociation ou d'un pouvoir de marché qui serait absolu; la détermination du salaire réel, donc le partage de la valeur ajoutée, est le résultat du conflit de répartition, il s'agit d'un compromis. Il est stable dès lors que le taux d'inflation (le taux de croissance 
des prix) est équivalent au taux de croissance des salaires nominaux ${ }^{5}$. Les modalités d'obtention d'un tel compromis (ou de l'impossibilité de son obtention) peuvent faire l'objet d'une analyse d'économie politique historique. Une telle démarche appliquée au cas argentin est proposée au sein de la section 2.

\section{2. En régime de haute inflation, des mécanismes d'indexation se développent} situation pouvait par exemple être observée au sein des pays développés lors des années 1970, mais aussi et surtout au sein de nombreux pays latino-américains jusqu'à la fin des années 1980. L'économiste structuraliste brésilien Celso Furtado expliquait dès 1958 que l'inflation observée au Brésil était la conséquence de revendications incompatibles quant à la répartition fonctionnelle des revenus. Noyola Vázquez (1956) souligne de son côté que la rigidité de l'offre peut renforcer une tendance à l'accélération de l'inflation (c'est l'inflation structurelle).

Un élément nous apparaît essentiel pour notre analyse: si les auteurs structuralistes construisent une théorie de l'inflation aux fondements similaires à la théorie postkeynésienne, les auteurs structuralistes à partir des années 1980 (les néostructuralistes) vont particulièrement s'intéresser aux mécanismes institutionnels qui accroissent ou entretiennent une trajectoire inflationniste. Ces auteurs expliquent que les comportements des agents vont évoluer lorsqu'ils intègrent le fait que l'inflation peut varier brusquement, lorsqu'elle est habituellement forte ou lorsqu'elle tend à se renforcer. Nous souhaitons intégrer cet élément à notre réflexion.

Roberto Frenkel (1979) a ainsi dévoilé l'importance de l'indexation dans les régimes de haute inflation. Il explique que si l'inflation est forte et volatile sur plusieurs périodes, les agents vont chercher à développer des mécanismes d'indexation afin de protéger leurs revenus réels contre les effets provoqués par une éventuelle accélération de l'inflation. Ce mécanisme institutionnel entretient naturellement l'inflation, mais il est en outre susceptible d'accélérer l'inflation (voir aussi Taylor, 2004). Carvalho (1993) écrit d'ailleurs que l'indexation renforce le sentiment de sécurité des agents dans un environnement rendu encore plus incertain par l'inflation. Ces mécanismes génèrent une forte inertie dans la trajectoire inflationniste, d'où le terme parfois utilisé d'inflation inertielle. Câmara Neto et Vernengo (2001) soulignent que les auteurs néostructuralistes ont pu largement insister au sein de leurs recherches sur les causes de l'inflation dite inertielle. Les comportements d'indexation sont alors les principaux responsables de l'accélération et de la diffusion des hausses de prix. De notre point de vue, intégrer ces éléments ne doit pas se faire au détriment de l'analyse et de la compréhension de la difficile résolution du conflit de répartition. En d'autres termes, il ne suffit pas de supprimer toute indexation dans une économie qui évolue sous un régime de haute inflation pour espérer que l'inflation redevienne durablement faible, car le conflit de répartition ne tardera pas à générer à nouveau de l'inflation. L'échec en Argentine du plan Austral (cf. section 2) illustre particulièrement cet aspect.

L'indexation doit être considérée comme une construction institutionnelle qui est provoquée par un processus inflationniste violent et durable au sein de l'économie. Les phénomènes d'indexation viennent alors s'ajouter au conflit de répartition, qui peut peiner à faire émerger un compromis stable et faiblement inflationniste sur le partage

Revue de la régulation, 15 | 1er semestre / Spring 2014 
de la valeur ajoutée. Carvalho (1991) distingue le régime d'inflation modérée du régime de haute inflation à l'aune du critère de l'indexation. Il estime qu'une inflation annuelle proche de $20 \%$ nécessite la création de mécanismes d'indexation. Comme l'auteur le reconnaît, l'indexation crée elle-même ses propres vulnérabilités, notamment dans la propagation d'une rapide et forte accélération de l'inflation. Dans le cas où l'évolution du taux de change devient un élément important dans les comportements et l'indexation, la dynamique peut alors provoquer l'hyperinflation. C'est la proposition développée dans cet article. Le conflit de répartition restant bien sûr à l'origine de la trajectoire inflationniste.

\section{3. Anticipations sur le change et fuite devant la monnaie : la possibilité de l'hyperinflation} générée par le conflit de répartition qui oppose capitalistes et travailleurs et qui porte sur le partage de la valeur ajoutée. Lorsque l'inflation est violente et durable, émergent des phénomènes d'indexation des prix et des salaires qui tendent à l'entretenir et la renforcer. Si l'inflation se renforce ainsi, cela signifierait-il qu'il est possible de repérer l'hyperinflation avec un critère quantitatif ? Devons-nous nous résoudre à accepter le critère purement quantitatif de Cagan ou à discuter son niveau?

Nous proposons un critère alternatif, qualitatif. Nous estimons que l'hyperinflation diffère de l'inflation, même dans son expression violente. L'hyperinflation a une nature différente : c'est le rejet de la monnaie domestique. Les épisodes d'hyperinflation sont observés lorsqu'une monnaie a successivement perdu ses trois fonctions cardinales (unité de compte, moyen de paiement, réserve de valeur) au profit d'une devise. La préférence pour la liquidité est susceptible d'expliquer ce phénomène. Les agents recherchent l'utilisation d'un actif liquide et dont la valeur est stable. Généralement, c'est la monnaie domestique qui remplit ce rôle. Mais si celle-ci n'offre plus ces conditions, les agents recherchent des substituts, comme rappelé par Davidson (1992) ou Dow (1999, p. 154-155):

Within a domestic economy, the national currency is generally the asset which is most liquid and most stable in value. But within the international economy, there is a range of moneys. As long as each has a stable value in relation to the others, then the most liquid of these is generally employed as a means of payment and unit of account; this would normally be the national currency of the economy concerned. But, where the domestic value of the national currency is falling significantly relative to foreign currencies, because of domestic inflation or a depreciating exchange rate, other currencies may better satisfy liquidity preference. [...] In cases of hyperinflation, capital controls may not be sufficient to prevent demonetization and the substitution of foreign currency for domestic currency.

Adoptant cette vision, nous retenons que l'hyperinflation est une violente inflation qui va de pair avec la substitution de la monnaie domestique par une devise. Il y a donc bien une relation avec le marché des changes à explorer. Robinson (1966) explique que l'hyperinflation allemande est initiée par l'existence d'un cercle vicieux inflation dégradation du taux de change réel - augmentation de l'endettement externe en devises - dévaluation (ou dépréciation externe de la monnaie) - renforcement de l'inflation, ce qui mène à nouveau au début de l'enchaînement causal. L'importance des paiements externes dans une dynamique hyperinflationniste est aussi un élément mis en exergue par Câmara Neto et Vernengo (2001). Ils soulignent que les auteurs néo- 
structuralistes semblent avoir négligé, dans leurs analyses des processus hyperinflationnistes, le fait qu'ils trouvent leurs origines dans le paiement de dettes externes et dans l'obligation de déprécier la monnaie domestique afin d'obtenir des surplus commerciaux.

Face à une telle situation, nous estimons que les agents peuvent progressivement développer des anticipations relatives à une prochaine dévaluation (si la Banque centrale s'est engagée dans un plan de stabilisation du change) ou de façon générale sur la variation du change. De telles anticipations peuvent donner naissance à des comportements autoréalisateurs. Les agents sont susceptibles de provoquer un run sur les réserves de change, ce qui déclenche le lâchage du change et par là-même l'hyperinflation telle que définie précédemment (les agents cherchent alors à substituer le plus rapidement possible des unités de monnaie domestiques en devises). Les anticipations sur le change forment donc le troisième élément constitutif de l'hyperinflation, après le conflit de répartition et l'indexation. Autrement dit, la théorie post-keynésienne semble pouvoir défendre la position théorique suivante: l'hyperinflation se développe au sein d'une économie endettée en devises et qui connaît un déficit du solde courant de la balance des paiements. Dans cette économie, on observe un conflit de répartition virulent, auquel s'ajoutent des phénomènes d'indexation et d'anticipations sur le change.

Cette séquence va maintenant être confrontée aux faits observés en Argentine dans les années 1970-1980. On va donc chercher à étudier l'évolution du conflit de répartition, la mise en place de mécanismes d'indexation et une éventuelle fuite de la monnaie domestique vers une devise, simultanément au déclenchement de l'hyperinflation.

\section{Application au cas argentin}

\section{1. Un conflit de répartition virulent dès les années 1970}

L'inflation connaît, en Argentine, une rapide accélération au milieu des années 1970. À partir de cette période, et à la faveur d'une inflation volatile mais généralement très importante, le partage de la valeur ajoutée entre revenus du capital et revenus du travail va connaître une grande variabilité, comme l'indique le graphique suivant qui présente l'évolution de la part des salaires dans le PIB. Ainsi, la part des salaires peut passer de $49,69 \%$ en 1974 (plafond historique) à $29,74 \%$ en 1977 , tout comme elle évolue nettement entre 1986 et 1988, passant de $41 \%$ à $33 \%$, et ce avant même que ne se déclenche l'hyperinflation. 
Graphique 1. Part des salaires dans le Pib (1970-1989)

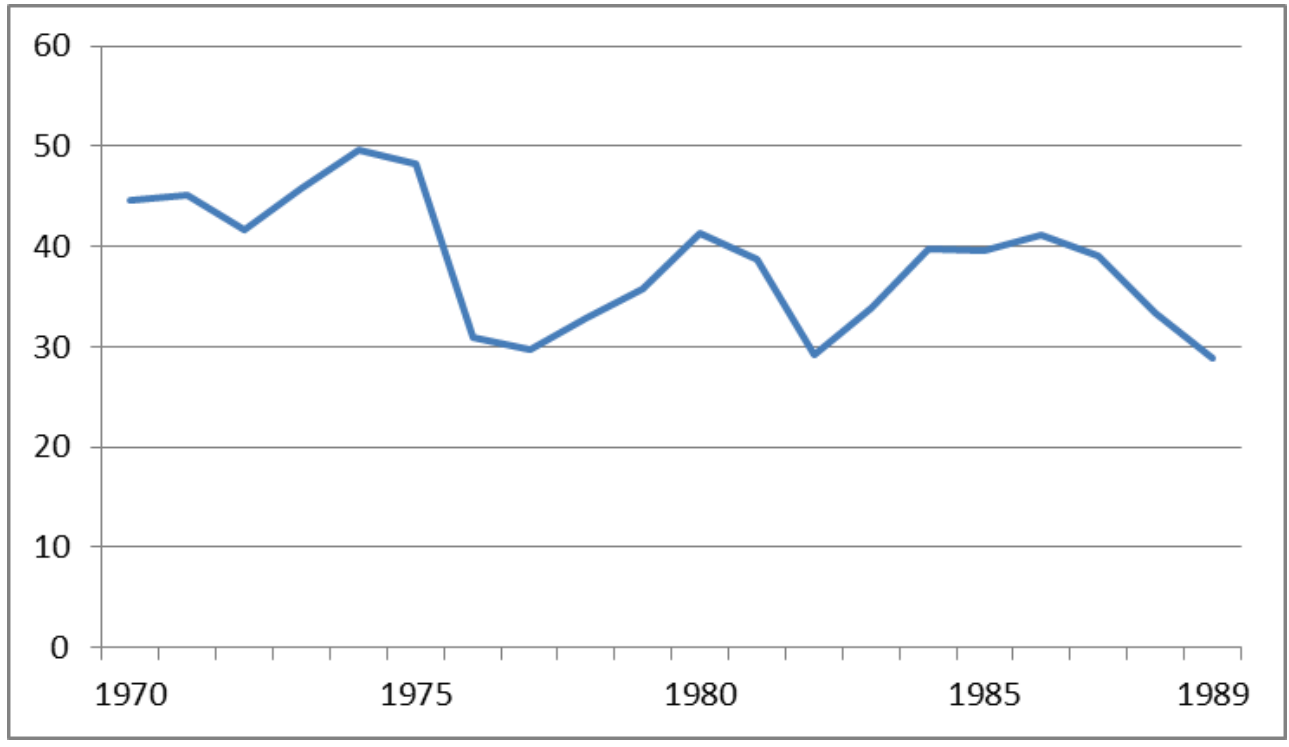

Source : D’après Graña et Kennedy (2008), sur des données réelles

Toutefois, l'objectif de cet article n'est pas d'étudier l'évolution de la répartition du revenu. Il s'agit de comprendre l'évolution de l'inflation jusqu'à l'hyperinflation observée en 1989. Certes, ces questionnements sont connexes (puisque la variation brutale de l'inflation peut provoquer un changement rapide dans la répartition du revenu), mais ces problématiques sont malgré tout différentes.

Nous proposons de débuter l'analyse spécifique en 1973 pour deux raisons. La première, parce que les statistiques officielles (fournies par l'INDEC) indiquent que l'inflation (prix à la consommation, en rythme annuel) passe de 40,1\% à 347,5\% entre 1973 et $1976^{6}$. La seconde, parce qu'il est possible d'appréhender cette évolution à travers l'analyse post-keynésienne du conflit de répartition (Marie, 2010). La période est alors marquée par la conjonction de deux éléments : d'une part un pouvoir de marché des firmes important et qui se renforce sous l'effet de la croissance économique, et d'autre part un pouvoir de négociation des travailleurs lui aussi très important (les taux de chômage sont faibles) et favorisé par le retour au pouvoir de Juan Perón. Notons que le contexte politique tendu ne permet pas d'envisager un apaisement à court terme du conflit de répartition.

En mars 1976, un coup d'État militaire renverse le pouvoir constitutionnel ${ }^{7}$. La situation économique est marquée par le renforcement de l'inflation (cf. graphique 2) mais aussi par le faible niveau des réserves de change. L'hypothèse d'un défaut de paiement sur la dette publique apparait comme l'une des raisons du coup d'État (Calvo, 1986; Schvarzer, 1987). Les militaires resteront au pouvoir jusqu'à la fin du premier trimestre 1983. Quel a été l'impact de ce changement de régime politique sur la politique économique menée ? On s'intéresse bien sûr au conflit de répartition et à l'inflation.

La politique économique des militaires rompt avec celle menée précédemment. Les contrôles sur les prix sont supprimés et le système de change est simplifié (un taux de change unique est dorénavant appliqué à tous les échanges extérieurs). Ces décisions de libéralisation de l'économie s'accompagnent immédiatement d'un soutien financier international. Le 31 mars 1976, les réserves de change s'élèvent grâce à l'octroi d'un prêt de 100 millions de DTS de la part du FMI. La position est consolidée par un nouveau 
prêt de 260 millions au mois d'août. Ces prêts diminuent nettement la probabilité d'un défaut de paiement.

Mais la rupture la plus spectaculaire concerne la politique salariale. Bénéficiant d'un important pouvoir de coercition (outre la féroce répression politique, l'activité syndicale est encadrée et le principal syndicat, la CGT, est dissout), le ministre de l'Économie impose en avril un gel des salaires nominaux pour une durée de 3 mois. Cette décision provoque une modification importante et quasi-immédiate de la répartition du revenu au sein de l'économie argentine que l'on peut remarquer sur le graphique 1. Comme le précise Frenkel (1984), les salaires réels diminuent de $35 \%$ en un trimestre, et leur niveau aura été durablement affecté. Nous pourrions ajouter que la répartition fonctionnelle des revenus a été tout aussi durablement réorientée en défaveur des revenus salariés. Cependant, l'inflation observée (graphique 2) diminue à la faveur de cette politique. C'est la conséquence de la diminution du pouvoir de négociation des travailleurs. Cette modification des termes du conflit de répartition a des conséquences macroéconomiques; la diminution du salaire réel provoque une diminution de la consommation qui est compatible avec la récession observée en 1976.

Graphique 2. Taux mensuels d'inflation, de janvier 1976 à décembre 1981

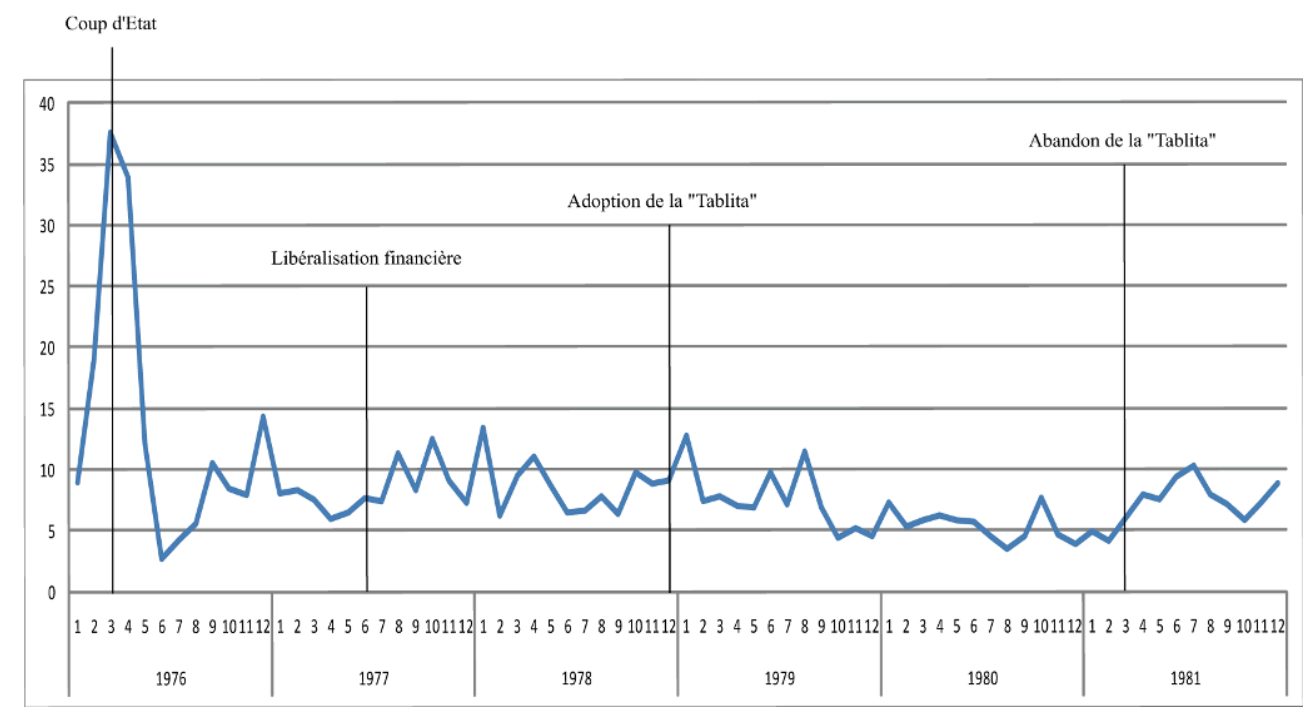

Source : Indice IPC, INDEC

L'inflation, erratique, va cependant suivre à nouveau une tendance au renforcement au cours du second semestre de l'année 1977. Selon de nombreux auteurs, la libéralisation financière est l'objectif de la politique économique établie par les militaires; cet argument est notamment développé par Canitrot (1980), Frenkel (1980) ou encore Schvarzer (1987). Or, le $1^{\text {er }}$ juin 1977 interviennent les lois sur la décentralisation des crédits et sur la libéralisation des institutions financières (indiquées sur le graphique 2). Les taux d'intérêt bancaires connaissent une forte augmentation. Alors qu'ils étaient nuls en termes réels, ils deviennent nettement positifs : le taux d'intérêt moyen débiteur à 30 jours passe de 7,4\% en juin 1977 à 13,6\% en décembre alors que l'inflation observée ce même mois est de 7,3 \% (ADEBA, 1977). L'augmentation soudaine des taux d'intérêt déclenche une brusque récession et une accélération de l'inflation qui se renforcent conjointement lors du premier semestre 1978 (le taux de croissance du PIB avait été nettement positif en 1977). 

cette situation stagflationniste ${ }^{8}$. Les coûts auxquels sont confrontées les entreprises augmentant, celles-ci peuvent chercher à accroître leur taux de marge sur les salaires et la cible de salaire réel formulée par les entreprises diminue (le taux de marge visé s'élève). C'est bien ce que l'on observe : au cours du second semestre 1977, la hausse des taux d'intérêt implique une augmentation des coûts financiers des entreprises. L'inflation progresse et le salaire réel diminue. Ces éléments sont cohérents avec les statistiques (mais aussi avec Frenkel, 1984 ou Basualdo, 2006). paramètres du conflit de répartition (Lavoie, 1992, p. 404). Le pouvoir de négociation des travailleurs ne semble pas être une variable sur laquelle le gouvernement peut jouer à nouveau. Les cibles de salaires ou de taux de marge ne sont pas des éléments qui peuvent être affectés à court terme par la politique économique menée. Cependant, il est possible de réduire le pouvoir de marché des entreprises, en les exposants à une concurrence internationale accrue. Cet objectif va être poursuivi par la junte militaire, à travers la mise en place d'une libéralisation externe de l'économie appuyée par une politique de change particulière.

Si la politique de change menée jusqu'alors vise à maintenir constant le taux de change réel (Calvo, 1986), elle est profondément modifiée en décembre 1978, avec la mise en place de la Tablita9 (événement inscrit sur le graphique 2). Dans un contexte d'ouverture des transactions financières et commerciales, les taux de dévaluation sont annoncés à l'avance et décroissants de façon linéaire. Les taux initiaux sont nettement inférieurs aux taux d'inflation observés (respectivement de 5,4\% et $9 \%$ en rythme mensuel pour janvier 1979, contre environ $1 \%$ d'inflation aux États-Unis).

Cette politique, menée de décembre 1978 à décembre 1980 (son abandon officiel intervient en mars 1981) engendre plusieurs effets remarquables sur la macroéconomie argentine. L'inflation diminue à la faveur de la politique menée. De $169,8 \%$ en en rythme annuel pour 1978, elle diminue à $139,7 \%$ en 1979 puis à $87,6 \%$ en 1980 . Seulement, malgré cette diminution de l'inflation, le taux de change réel des devises en pesos diminue, provoquant certes l'effet disciplinant recherché, mais dégradant aussi très nettement le solde commercial par la perte de compétitivité des produits argentins dans un contexte d'accroissement des échanges extérieurs. Le solde des transactions courantes de la balance des paiements passe ainsi d'un solde positif de 1833,6 millions de dollars en 1978 à un solde négatif de 4768 millions de dollars en 1980 (données de la Banque centrale reprise par CEPAL, 1986, p. 342). Ce déficit courant s'accompagne d'un nécessaire accroissement des entrées de capitaux.

Grâce à un différentiel de taux d'intérêt favorable aux entrées de capitaux (selon la Fed le taux d'intérêt créditeur à 30 jours est de $0,79 \%$ en janvier 1979 aux États-Unis contre $6,8 \%$ en Argentine selon l'ADEBA ${ }^{10}$ ), à une situation de grande liquidité des marchés financiers internationaux et à la politique d'ancrage du change (qui promet un risque de change nul), l'Argentine parvient à attirer instantanément une quantité très importante de capitaux. Le solde des crédits financiers au sein de la Balance des Paiements passe ainsi de 1482 millions de dollars à 3643 millions en 1979 et reste nettement positif en 1980. Ces crédits, en grande partie bancaires (ils apparaissent sous l'intitulé "crédits financiers» au sein des statistiques de balance de paiement reportées en annexe), se font à des échéances brèves. Cependant, au cours de l'année 1979, les taux d'intérêt externes augmentent sous l'impulsion du changement de 
politique monétaire mené par la Fed. Par répercussion, les taux d'intérêt s'élèvent aussi en Argentine. Dans ces conditions, une crise financière se développe dès la fin du premier semestre 1980. Cette crise de solvabilité de nombreux acteurs financiers est provoquée par les multiples défauts de paiement d'agents endettés lors des années précédentes (Miotti, 1995). La Banque centrale est alors contrainte d'effectuer une intervention de prêteur en dernier ressort pour alimenter en liquidité les établissements bancaires. Les entrées de capitaux de la fin de l'année 1980 puis de 1981 s'effectuent à des taux d'intérêt toujours croissants et la crédibilité du système est mise en doute. La politique d'ancrage du change est ainsi abandonnée en mars 1981.

41 Celle-ci, combinée à l'ouverture financière, n'a pas permis d'instaurer la stabilité. Certes, l'inflation a pu être réduite sans remettre en cause le processus de libéralisation financière dans lequel s'était engagé le gouvernement militaire. Seulement, la politique suivie a instillé un élément d'instabilité d'origine externe. Début 1981, l'économie argentine souffre d'un niveau d'endettement externe qui s'est très nettement accru, et comme nous le verrons dès la section suivante, les comportements des agents argentins ont été durablement modifiés.

\section{2. La présence de mécanismes d'indexation}

Les années 1980 ne permettent pas d'obtenir un compromis stable issu du conflit de répartition. Les termes de celui-ci sont à nouveau régulièrement modifiés, la faiblesse de l'investissement empêche l'accroissement des capacités de production et l'obtention de forts gains de productivité. L'inflation se renforce tendanciellement pendant que des mécanismes d'indexation se mettent en place, entretenant la dynamique inflationniste. De plus, la « décennie perdue » est marquée par la nécessité d'obtenir via le commerce extérieur les devises nécessaires au respect des engagements financiers externes, contrainte supplémentaire pesant sur l'économie argentine.

La Tablita abandonnée, plusieurs dévaluations sont menées au cours des années 1981 et 1982. Le taux de change nominal du dollar en peso est multiplié par près de 27 entre mars 1981 et juin 1982, multipliant le taux de change réel bilatéral avec les États-Unis par 4,8 (Martinez, 2003). Le solde du compte commercial passe d'un déficit de plus de 2,3 milliards de dollars courants en 1980 à un excédent de 2,3 milliards en 1982. Cette politique est rendue nécessaire par le solde négatif croissant des services financiers (qui passe de 1,5 milliards en 1980 à 4,7 en 1982) alors que les entrées de capitaux sont de plus en plus difficiles depuis le défaut de paiement du Mexique sur sa dette externe en 1982.

Ces aspects renvoient à la gestion de la dette externe, en grande partie provoquée par un endettement externe privé développé à la faveur de la Tablita ${ }^{11}$. Sur un plan interne, avec la diminution de la valeur de la monnaie nationale, le poids des dettes contractées en dollars s'élève pour les débiteurs qui ont des ressources en monnaie nationale. L'État argentin permet la transformation d'une partie de la dette privée externe en une dette interne dont l'État est le créancier. C'est une nationalisation de la dette externe (pour le détail de ce processus, on peut proposer de se référer à Damill et al., 2005 ou Basualdo, 2006, p. 218). Depuis 1981, l'inflation progresse à nouveau. Si l'inflation annuelle s'établissait à 87,6 \% en 1980, elle atteint $131,3 \%$ en $1981,209,7 \%$ en 1982 et $433,7 \%$ en 1983. Interrogeons-nous sur l'ampleur de cette augmentation. 

pouvoir de marché des entreprises se trouve dorénavant renforcé par la politique de change. En 1983 et principalement à la faveur d'un contexte politique différent (la déliquescence du pouvoir autoritaire), le pouvoir de négociation des travailleurs s'élève provoquant une élévation des salaires réels. Soulignons qu'en concordance avec l'analyse post-keynésienne, cette augmentation des salaires réels s'accompagne d'une augmentation du PIB (celui-ci progresse de $2,6 \%$ en 1983). Nous observons un renforcement conjoint du pouvoir de marché et du pouvoir de négociation.

Face à ces reformulations récurrentes des termes du conflit de répartition, les agents développent des mécanismes d'indexation pour se prémunir contre ces variations brutales des prix qui entrainent des modifications immédiates des revenus réels. Frenkel (1984) indique que, depuis la fin de l'année 1976, l'évolution des salaires nominaux a tendance à s'indexer sur le niveau de l'inflation observé lors de la période précédente. D'ailleurs, l'indexation n'est pas nécessairement formelle :

L'Argentine connaît à la fois des mécanismes d'indexation formelle, surtout dans le secteur privé, et une indexation informelle qui se manifeste, dans le secteur public, sous l'aspect spécifique d'une «routinisation » des conflits, comme forme sociale d'adaptation à l'inflation: de nombreux conflits se déclenchent en effet de façon relativement régulière, pour récupérer précisément le pouvoir d'achat amenuisé par l'inflation (Salama et Valier, 1990, p. 86).

L'indexation observée en Argentine n'est pas exclusivement de type adaptatif (ou backward looking) et n'est pas appliquée uniquement aux revenus du travail. Fanelli et Chisari (1989) estiment qu'il existe deux éléments sur lesquels se concentre l'indexation : les tarifs publics et le taux de change. Au moment de nouer les contrats ${ }^{12}$, firmes et travailleurs s'accordent pour indexer les salaires sur les variations des tarifs publics et les variations du change, à charge pour les firmes de répercuter cette indexation sur les prix. L'accord est rendu possible par le fait qu'il ne va engendrer de modification ni du salaire réel, ni du taux de marge des firmes, et qu'il réduit drastiquement les coûts de transaction.

Comment comprendre l'intégration des variations du taux de change? Les comportements ont été grandement influencés par la Tablita. Le mécanisme de ce régime de change reposait sur l'annonce anticipée des taux de dévaluation du peso face au dollar. Cette politique de change, adoptée dans un contexte de libéralisation financière, a naturellement encouragé l'utilisation du dollar. Elle a encouragé les agents à prendre appui sur cette devise comme réserve de valeur et comme unité de compte simultanément à l'utilisation du peso, la monnaie domestique. Sgard (2007), dans une comparaison entre les ordres monétaires brésilien et argentin des années 1980, indique justement que l'ordre monétaire influence les comportements. Il souligne judicieusement l'importance de la politique monétaire menée pour saisir l'importance du dollar dans les comportements économiques argentins. Les agents économiques ont ainsi été particulièrement confrontés au rôle déterminant de l'évolution du taux de change dans une économie inflationniste. D'autre part, pour les agents endettés en devises, la conséquence d'une dévaluation sur les passifs peut être très douloureuse quand les ressources sont en monnaie domestique. Les firmes endettées doivent donc être attentives aux variations du taux de change; une dépréciation de la monnaie domestique renchérit le coût de l'emprunt contracté en devises. Si le raisonnement est valable pour les passifs, il l'est aussi pour les actifs. Le dollar devient l'actif de réserve de valeur; on évalue sa richesse en unités de cette devise. C'est donc bien un début de

Revue de la régulation, 15 | 1er semestre / Spring 2014 
dollarisation auquel on assiste. De nombreux agents ont un intérêt commun à voir le taux d'inflation ou le taux de croissance des salaires nominaux suivre l'évolution du change, afin de limiter les pertes de leur revenu exprimé en dollars. Dès lors, lorsque de brutales variations du taux de change sont observées, le taux d'inflation interne réagit instantanément. Ces éléments sont cohérents avec la réflexion théorique menée au sein de la section 1.

Lorsque le taux de change augmente de plus de $200 \%$ en juillet 1982, le taux d'inflation mensuel dépasse $16 \%$ (alors qu'il était de moins de $8 \%$ le mois précédent). La dynamique profonde de l'inflation doit toujours être recherchée dans l'évolution des termes du conflit de répartition (on a relevé une augmentation du pouvoir de négociation des travailleurs alors que le pouvoir de marché reste très élevé), mais intégrer les phénomènes d'indexation nous permet de saisir pourquoi peuvent survenir des pics inflationnistes indépendamment de changements notables dans les paramètres du conflit de répartition. Soulignons enfin que l'évolution de la productivité ne permet pas d'affaiblir franchement le rythme inflationniste; la productivité du travail progresse certes de 3,6\% en 1981 (Frenkel, 1984), mais elle recule de près de $4 \%$ en 1982 (Coremberg et al., 2007). Et si en 1983 on observe une nouvelle progression de $5 \%$, par la suite, la productivité recule chaque année jusqu'en 1989 (sauf en 1987; elle progresse alors de moins de $1 \%$ ). Ces variations sont de toute façon sans commune mesure avec le rythme puis le renforcement du taux de croissance des prix.

\section{3. Les conditions de l'hyperinflation révélées par les échecs des plans Austral et Primavera}

La restauration de la démocratie et l'élection de Raul Alfonsin en 1983 ne permettent pas de lever les fortes contraintes pesant sur l'économie. Dans la sous-section 1. 3., nous avons proposé que l'hyperinflation se manifeste simultanément à une très forte dépréciation externe de la monnaie, celle-ci pouvant être provoquée par des comportements autoréalisateurs. C'est l'existence d'une telle séquence que nous souhaitons maintenant vérifier, et celle-ci se repère dans le double échec de plans de stabilisation, celui du plan Austral (1985) puis celui du plan Primavera (1988).

51 Suite aux élections de 1983, le gouvernement tente d'imposer une renégociation de la dette publique externe (en 1984, selon les statistiques de balance des paiements, les exportations argentines représentent environ 4200 millions de dollars et les paiements versés au titre des obligations financières avec l'étranger 6000 millions de dollars). Cette position s'appuie sur la suspension de tous les versements au titre du principal et sur le retard systématique des versements au titre des intérêts sur la dette publique (Smith, 1991 ; Heredia, 2006). Cependant, le pays reprend intégralement ses paiements d'intérêts en septembre 1984 et signe un accord avec le FMI en décembre.

Concernant la gestion interne, le gouvernement permet une augmentation des salaires en décembre 1983. Des règles devant encadrer l'évolution future des prix industriels, des tarifs publics, du taux de change et des salaires sont instaurées alors qu'un taux d'intérêt régulé est établi à $11 \%$ en janvier 1984. Ce taux correspond au taux d'inflation visé par le gouvernement. Heymann (1986, p. 145) mentionne que cette politique de règles était intenable : les prix relatifs (entre les biens dont les prix sont contrôlés et ceux qui ne le sont pas) pouvaient se voir désarticulés dans des proportions importantes à très court terme. Le gouvernement est dans le même temps confronté à 
l'opposition des entrepreneurs concernant les augmentations de salaires nominaux (qui se sont transformées en augmentations des salaires réels renforçant la tendance observée en $1983^{13}$ ) et au rejet de toute réforme des impôts. Selon Smith (1991), une stratégie d'opposition systématique est aussi adoptée par la CGT, principale centrale syndicale, ainsi que par les partis d'opposition. Enfin, le «Campo", le secteur agroexportateur, rejette les taxes sur les exportations.

Le conflit de répartition apparait toujours aussi vif. De plus, lors de l'année 1984, la croissance économique ralentit et le solde de la balance commerciale qui était nettement positif lors des deux premiers trimestres de l'année est à peine positif lors du dernier trimestre. Le FMI exige un nouvel ajustement du taux de change, c'est-à-dire la mise en place d'une dévaluation compétitive. Le gouvernement se conforme à cette recommandation à la fin de l'année (le taux de change nominal du dollar en pesos est multiplié par 2,7 entre septembre 1984 et février 1985). Favorisé par les mécanismes d'indexation, le taux d'inflation annuel atteint $688 \%$ en 1984 et le rythme s'accélère encore en fin d'année. Lors du premier semestre 1985 , les prix à la consommation augmentent de plus de $20 \%$ chaque mois et le taux de chômage s'élève $(3,5 \%$ en octobre 1984, 5,7\% en mai 1985). Pour tenter de mettre fin à cette spirale, le plan Austral est adopté en juin.

Ce plan repose sur trois piliers (Heymann, 1987). D'abord un pilier fiscal. Le gouvernement escompte une amélioration du solde budgétaire par l'augmentation des droits sur les exportations, par celle des tarifs publics mais aussi grâce à un effet "Olivera-Tanzi » inversé (si l'inflation diminue, le rendement des impôts sera meilleur en termes réels) et par une réforme de la collecte des impôts directs. Deuxième pilier de la mesure, le plan introduit un gel des prix et des salaires. Seuls certains prix sont laissés libres, comme ceux des fruits et légumes, pour lesquels on estime que les producteurs sont preneurs de prix. Un taux de change officiel appliqué au commerce extérieur est établi selon le gouvernement à un niveau adéquat pour stimuler la promotion des exportations du pays et doit être figé à la valeur de 0,80 unité de monnaie domestique pour un dollar. Des taux d'intérêt régulés sont mis en place. Les taux nominaux pour les opérations à 30 jours sont annoncés à $3,5 \%$ pour les taux créditeurs et $5 \%$ pour les taux débiteurs alors que les taux en vigueur lors de la première quinzaine de juin étaient de l'ordre de $28 \%$, toujours pour un dépôt à 30 jours (BCRA, 1987, p. 45). Troisième et dernier pilier sur lequel repose le plan, celui du changement du signe monétaire : l'Austral remplace le Peso (avec une conversion de 1 Austral pour 1000 pesos). En créant un nouveau signe monétaire, on cherche à reconquérir à partir de la fonction d'unité de compte les autres fonctions cardinales de la monnaie. Cela peut s'interpréter comme une tentative de rupture des liens internes «parasites» entre la monnaie nationale et son concurrent, le dollar (Sgard, 2007, p. 471).

55 Ces réformes apparaissent comme une franche réussite à court terme ${ }^{14}$. Les taux mensuels d'inflation observés lors du second semestre de 1985 sont tous inférieurs à $5 \%$. Le programme de désindexation (appuyé par une échelle de conversion des dettes qui intègre un taux mensuel d'actualisation d'environ $23 \%{ }^{15}$ ) apparaît efficace, tout comme les efforts entrepris pour permettre la formulation de nouvelles anticipations (Canavese et Di Tella, 1991, p. 159). L'écart entre le taux de change officiel et le taux de change libre se réduit, de même qu'entre les taux d'intérêt régulés et les taux d'intérêt non régulés (BCRA, 1986, 1987). Le PIB progresse de 5,8\% en 1986, l'investissement 
progresse lui aussi malgré des taux d'intérêt réels positifs. Les anticipations des entrepreneurs évoluent favorablement, mais cela indique aussi que l'on demeure à un niveau élevé d'utilisation du capital et que les entreprises ont du mal à répondre à l'augmentation de la demande. D'ailleurs, dès 1986 on distingue une tendance au renforcement de l'inflation (graphique 3).

Graphique 3. Taux mensuel d'inflation, de janvier 1986 à décembre 1988

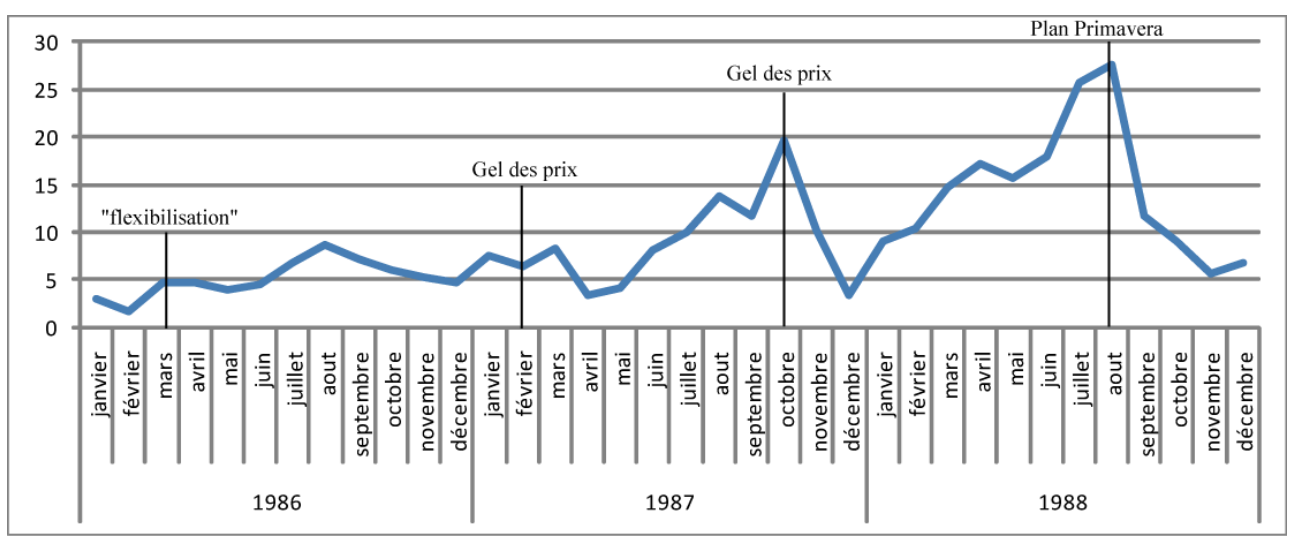

Source : Indice IPC, INDEC

Cette énième progression de l'inflation trouve ici son origine dans la levée des gels de prix en mars 1986 (notée flexibilisation sur le graphique 3). Le taux de change officiel évolue dorénavant de façon à maintenir constant le taux de change réel. Les prix doivent évoluer selon une règle négociée avec le gouvernement, ce dernier se portant garant du fait que les salaires réels restent constants. Canavese et Di Tella (1988) estiment que la flexibilisation des prix entraîne immédiatement une ré-indexation de l'économie. Heymann (1991) écrit quant à lui que celle-ci n'a en fait jamais disparu. Nous ajoutons que ce plan n'a pu offrir un compromis stable sur le partage de la valeur ajoutée.

57 Sur ces bases de stabilité fragile, un choc externe relance le processus inflationniste en 1987. Les termes de l'échange diminuent nettement; d'une base 100 en 1985 on atteint 84,9 en 1987 (Coremberg et al., 2007). Cet élément entraîne une chute du solde de la balance commerciale de 4,3 milliards de dollars en 1985 à 1,5 milliards en 1986. Les termes du conflit de répartition sont à nouveau affectés : l'augmentation du prix des biens importés a pu pousser les entreprises à augmenter leur cible de mark-up, comme les travailleurs leur cible de salaire réel. En janvier 1987 le taux d'inflation mensuelle est de 7,6\%, dépassant les objectifs du gouvernement (4\%). Celui-ci choisit de mettre en place un nouveau gel des prix et des salaires, reporté sur le graphique 3. En juin, lorsque s'achève ce gel, l'inflation se renforce immédiatement, atteignant $20 \%$ en octobre 1987. Conjointement, le salaire réel diminue, amorçant une tendance ininterrompue jusqu'en 1989 ('indicateur de salaire réel, repris de Damill et Frenkel, 1990, base $100=1985$, passe de 102,1 en 1986 à 96 en 1987, puis 91,3 en 1988 et 82,8 en 1989). Nous avons ici l'indication que le pouvoir de marché des entreprises relativement au pouvoir de négociation des travailleurs s'est renforcé.

La stabilisation permise par l'Austral est révolue. Les problèmes structurels sont toujours présents; le niveau d'endettement externe oblige l'Argentine à maintenir un solde commercial nettement positif, la structure économique argentine est affaiblie par 
les années de haute inflation et de faiblesse de l'investissement et enfin, la richesse financière apparaît toujours "dollarisée ». Le gouvernement est pris dans un cercle vicieux, confronté à son incapacité à s'endetter en émettant des titres libellés dans la monnaie nationale ${ }^{16}$. À la racine, toujours le conflit de répartition.

Le premier épisode hyperinflationniste se déclenche en mai 1989. Pour repérer le bouclage de la séquence hyperinflationniste post-keynésienne dévoilée dans notre première section, il faut prendre en compte ce que révèle l'échec d'un nouveau plan de stabilisation, celui du plan Primavera, adopté en août 1988 après plusieurs mois lors desquels l'inflation s'est nettement renforcée (graphique 3). Le programme est élaboré sur l'anticipation par les autorités que la nette tendance à la hausse des prix des denrées agricoles va se maintenir jusqu'à la fin de l'année. Les mauvaises récoltes estivales observées en Europe et aux États-Unis à la fin de l'été 1988 provoquent une hausse des cours, laissant espérer que les récoltes argentines du début de l'année 1989 encourageront de fortes entrées de devises, et amélioreront dans le même temps la balance fiscale par l'intermédiaire des taxes aux exportations. Alors que les réserves de change sont faibles, cette perspective explique la principale mesure du plan, qui introduit une modification du régime de change. Un taux de change dit «libre » est institué ; c'est un taux flottant, qui sera appliqué à toutes les importations et aux transactions financières. Un autre taux de change, situé à un niveau inférieur ${ }^{17}$ sera appliqué sur les exportations agricoles. Les autres exportations seront effectuées à un taux qui correspond à la moyenne entre ces deux taux de change. Cette décision est en fait un moyen de taxer les exportations et de reconstituer un stock de réserves de devises détenues par la Banque centrale, ce qui doit lui permettre d'intervenir sur le marché libre et de contrôler l'évolution du taux de change selon ses objectifs.

C'est dorénavant tout à fait clair : pour stabiliser l'inflation, il faut contrôler le taux de change. Initialement, grâce aux interventions de la Banque centrale, le taux de change «libre» est stabilisé, stabilisation qui se répercute sur le taux d'inflation (cf. graphique 4). Les variations du change sont effectivement primordiales dans les fluctuations rapides et violentes de l'inflation, comme l'indiquent les graphiques 4 et 5 . 
Graphique 4. Évolution du taux de change libre du dollar en Australs (taux de change nominal), janvier 1988 - décembre 1989

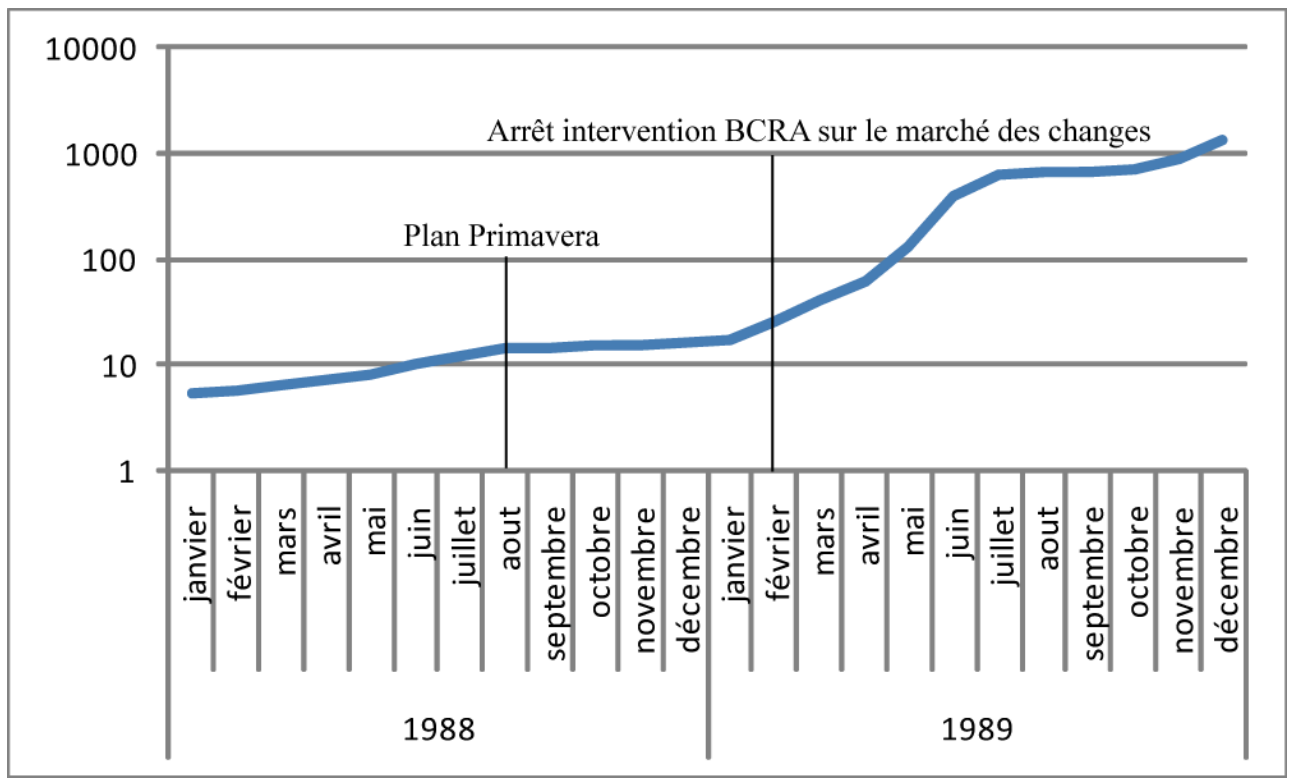

Source : BCRA

Graphique 5. Taux mensuel d'inflation, de juin 1988 à décembre 1989

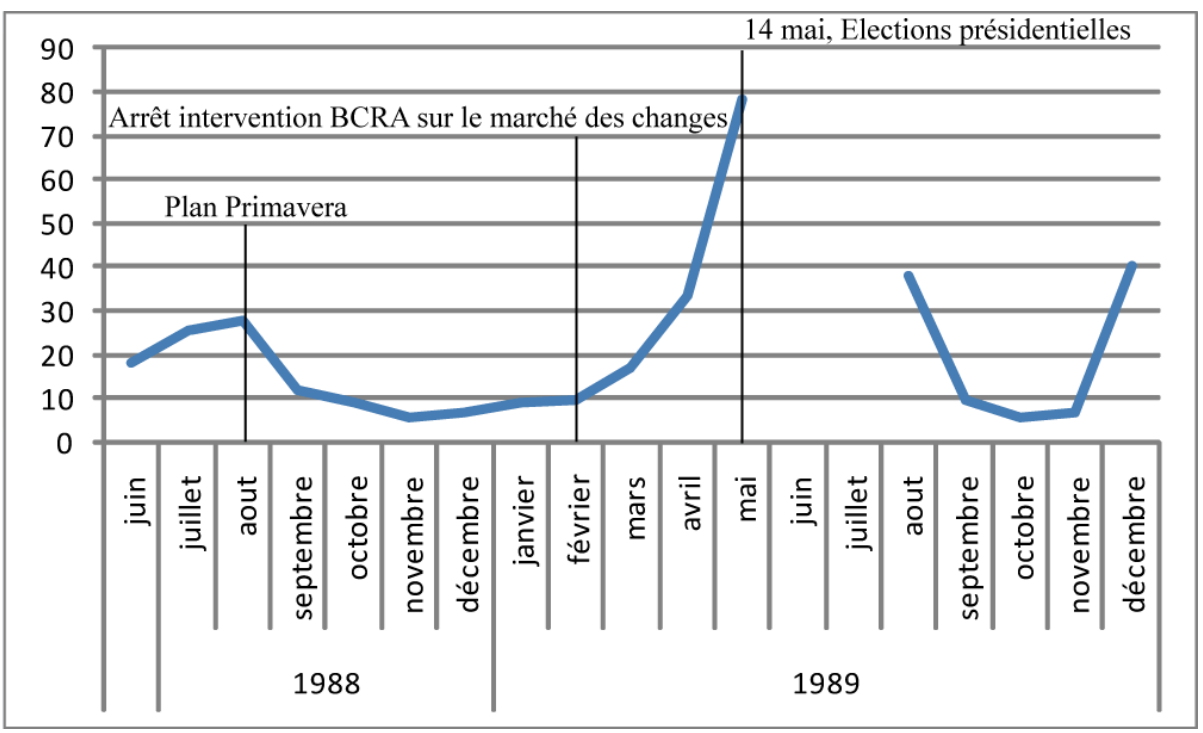

Source : Indice IPC, INDEC.

Note: Nous n'avons pas fait apparaître sur le graphique les taux d'inflation relevés pour les mois de juin et juillet 1989. La valeur de ceux-ci (respectivement 114,5\% et 196,6\%) était si élevée que les inscrire sur un même plan que les taux observés les autres mois rend la lecture plus difficile.

61 Les récoltes agricoles de la fin 1988 sont finalement très mauvaises. Selon Heymann (1991), confortant notre raisonnement concernant le déclenchement de l'hyperinflation, les anticipations sur le taux de change ont joué un rôle fondamental. Une masse de fonds prête à être changée s'est accumulée et un phénomène autoréalisateur se produit au tout début de l'année 1989, dans un contexte pré-électoral propice à l'instabilité ${ }^{18}$. La politique cherchant à contenir la progression du taux de change libre et à fournir un ancrage pour la valeur interne de la monnaie nationale est 
finalement sans issue. Les Argentins qui le peuvent transfèrent leurs actifs à l'étranger et se déclenche donc une course spéculative sur les réserves: la Banque centrale n'a plus la capacité d'intervenir sur le marché des changes à partir de février (graphique 4) alors qu'elle a vendu 900 millions de dollars la seconde quinzaine de janvier (Rapoport, 2007, p. 924). Dans ce contexte, l'inflation se renforce de manière incontrôlable, ouvrant la voie à l'hyperinflation.

Dorénavant, il s'agit d'obtenir le plus fort revenu en Australs avant d'effectuer les opérations de change, c'est-à-dire de se débarrasser le plus rapidement possible des Australs. Ce comportement engendre une course effrénée; seul le très court terme compte. C'est une fuite devant la monnaie. À travers la dollarisation et les difficultés perpétuelles rencontrées dans la résolution du conflit de répartition, a pu naître insidieusement l'idée que ce n'est plus la négociation en termes de monnaie nationale qui compte (l'Austral n'est utilisé que pour être aussitôt changé en dollars). L'évolution du conflit de répartition depuis 1976 a rendu possible l'hyperinflation. Les institutions capables de favoriser ou de provoquer l'obtention d'un compromis issu du conflit de répartition sont dorénavant trop affaiblies. On comprend qu'une crise hyperinflationniste s'accompagne d'une profonde crise politique.

\section{Conclusion}

L'article a proposé une interprétation compatible avec la théorie post-keynésienne concernant la survenue de l'hyperinflation en Argentine en 1989. Celle-ci est provoquée par un violent conflit de répartition, par des mécanismes d'indexation et par des anticipations sur le niveau de change. L'analyse de la trajectoire macroéconomique argentine des années 1970 jusqu'en 1989 a révélé que ce cadre théorique apparât pertinent. Sur la base d'un conflit de répartition violent, générant un processus inflationniste croissant et instable depuis les années 1970, des phénomènes d'indexation se sont développés et sont venus renforcer encore la pression inflationniste. Dans ce contexte, anticipant la rupture du change, la volonté des agents de substituer la monnaie domestique par une devise a finalement entraîné l'hyperinflation. La politique économique menée a pu encourager ces comportements pendant que les échecs de plans de stabilisation ont permis de révéler ce processus.

Bien sûr, l'accent mis dans cet article sur l'analyse de l'économie argentine ne doit pas nous faire oublier l'importance des facteurs externes sur cette trajectoire. L'endettement externe, qui s'est nettement accru à la fin des années 1970, a pu être constitué parce qu'on observait alors une abondante liquidité sur les marchés financiers internationaux. Inversement, les contraintes accrues dans le financement externe de l'Argentine des années 1980 sont intimement liées au resserrement de la politique monétaire aux États-Unis et à la crise de la dette déclenchée en 1982 par l'annonce du défaut de paiement du Mexique. Enfin, la difficulté à stabiliser le taux de change de la monnaie domestique vis-à-vis du dollar est d'autant plus forte que le marché des changes est lui-même profondément instable depuis l'abandon du système de Bretton-Woods. Ainsi, c'est la conjonction de l'instabilité de l'économie mondiale et de l'instabilité d'origine interne qui a rendu possible l'hyperinflation de 1989. Celle-ci, repérée simultanément à la réalisation d'anticipations concernant une forte dépréciation de la monnaie domestique vis-à-vis du dollar, est donc bien le rejet de la monnaie domestique au profit de cette devise. En d'autres termes, pour comprendre la 
survenue de l'hyperinflation, il nous a semblé que l'analyse du conflit de répartition était nécessaire mais non suffisante. Il fallait dans le même temps comprendre pourquoi le dollar avait une place si importante dans l'économie. Pour saisir ce phénomène, il est indispensable d'analyser le processus macroéconomique de moyen terme.

L'analyse proposée permet d'appréhender la raison pour laquelle l'Argentine fera le choix de se tourner vers un Currency Board en 1991, régime monétaire dont la monnaie de rattachement sera le dollar. L'échec de l'Austral a révélé l'incapacité à imposer une nouvelle monnaie aux agents argentins, ceux-ci préférant l'utilisation du dollar. Ce phénomène s'est clairement observé lors de l'hyperinflation de 1989, et fait écho à l'observation de Kaldor concernant l'hyperinflation allemande. Il relève ainsi (1982, p. 61) que : «[...] in Germany in September 1923, everything from newspapers to railway tickets and to daily wages was 'indexed' to the daily market price of the US dollar. [...] If the dollar remained unchanged for a day, prices and wages ... remained stable for the day".

Enfin, cet article ouvre des perspectives de recherche dans au moins deux directions. D'abord, bien sûr, dans la modélisation de tels processus. Mais aussi dans une seconde voie : il s'agirait de systématiser l'étude d'épisodes hyperinflationnistes dans l'histoire afin de vérifier que l'explication post-keynésienne proposée dans cet article peut être validée par d'autres épisodes. Les différentes trajectoires qui seraient analysées (y compris celles lors desquelles une haute inflation a pu être freinée durablement, comme par exemple en Israël au milieu des années 1980), devraient renforcer la réflexion théorique entamée ici.

\section{BIBLIOGRAPHIE}

ADEBA (1975-1982), Memoria Anual, Buenos Aires, Asociación DE Bancos Argentinos.

Aglietta M. et Orléan A. (2002), La monnaie, entre violence et confiance, Paris, Odile Jacob.

Arestis P. and Milberg W. (1994), "Degree of monopoly, pricing and flexible exchange rates", Journal of Post-Keynesian Economics, vol. 16, p. 167-188.

Basualdo E. (2006), Estudios de historia económica argentina, Buenos Aires, Flacso-Siglo Veinte Uno.

BCRA (1981-1987), Memoria Anual, Buenos Aires, Banco Central de la República Argentina.

Beccaria L. y Orsatti Á. (1985), "La evolución del empleo y los salarios en el corto plazo. El caso argentino, 1970-1983”, Documento de trabajo de la CEPAL de Buenos Aires, nº 14.

Blejer M. and Mathieson D. (1981), “The Preannouncement of Exchange Rate Changes as a Stabilization Instrument", IMF Staff Papers, vol. 28, p. 760-790.

Bresciani-Turroni C. (1937), The Economics of Inflation, Northampton, John Dickens.

Cagan P. (1956), “The Monetary Dynamics of Hyperinflation”, in Studies in the Quantity Theory of Money, Friedman M. (ed.), Chicago, Chicago University Press. 
Calvo G.A. (1986), “Fractured Liberalism: Argentina under Martinez de Hoz", Economic Development and Cultural Change, vol. 34, p. 511-553.

Câmara Neto A. and Vernengo M. (2001), “The German balance of payment school and the latin American Neo-Structuralists", in Credit, Interest Rates and the Open Economy, Rochon L.P. and Vernengo M. (eds.), Cheltenham, Edward Elgar, p. 143-159.

Canavese A. and Di Tella G. (1988), "Inflation Stabilization or Hyperinflation Avoidance? The Case of the Austral Plan in Argentine 1985-1987”, in Inflation Stabilization. The Experience of Israel, Argentina Brazil, Bolivia and Mexico, Bruno M., Di Tella G., Dornbusch R. and Fischer S. (eds.), Cambridge (MA), MIT Press, p. 153-190.

Canitrot A. (1980), “La disciplina como objetivo de la política económica. Un ensayo sobre el programa económico del gobierno argentino”, Desarrollo Económico, vol. 19, p. 453-475.

Carvalho F.J. (1991), “A Post Keynesian Approach to Inflation, High Inflation and Hyperinflation”, in Economic Problems of the 1990s, Davidson P. and Kregel J.A. (eds.), Cheltenham, Edward Elgar, p. 69-84.

Carvalho F.J. (1993), "Strato-Inflation and high inflation: the Brazilian experience", Cambridge Journal of Economics, vol. 17, p. 63-78.

Cassetti M. (2003), "Bargaining power, effective demand and technical progress: a Kaleckian model of growth", Cambridge Journal of Economics, vol. 27, p. 449-464.

Cassetti M. (2012), "Macroeconomic outcomes of changing social bargains. The feasibility of a wage-led open economy reconsidered”, Metroeconomica, vol. 63, p. 64-91.

CEPAL (1983), "Estadísticas económicas de corto plazo de la Argentina : precio, salario y empleo", Documento de trabajo, $\mathrm{n}^{\circ} 5$.

CEPAL (1986), "Estadísticas económicas de corto plazo de la Argentina : sector externo y condiciones económicas internacionales. Volumen I.", Documento de trabajo, $\mathrm{n}^{\circ} 20$.

Charles S. and Marie J. (2012), "Hyperinflation: a Post-Keynesian View”, AFEP-IHE-IPPE Conference, 5-8 juillet, Paris.

Coremberg A., Goldszier P., Heymann D. y Ramos A. (2007), "Patrones de la inversión y el ahorro en Argentina", Serie Macroeconomía del desarrollo, CEPAL, $\mathrm{n}^{\circ} 63$.

Dalziel P.C. (1990), "Market power, inflation and incomes policies”, Journal of Post-Keynesian Economics, vol. 12, p. 424-438.

Damill M. y Frenkel R. (1990), “Malos tiempos. La economía argentina en la década de los ochentas", Documentos CEDES, $\mathrm{n}^{\circ} 46$.

Damill M., Frenkel R. y Rapetti M. (2005), “La deuda argentina : historia, default y reestructuración”, Desarrollo Económico, vol. 45, p. 187-233.

Davidson P. (1992) [1982], International Money and the Real World, New York, MacMillan (2nd edition).

Dow S. (1999), "International Liquidity Preference and Endogenous Credit", in Foundations of International Economics Post-Keynesian Perspectives, Deprez J. and Harvey J.T. (eds.), London, Routledge.

Dutt A.K. (1990-1991), “Interest rate policies in LDCs: a Post-Keynesian view”, Journal of PostKeynesian Economics, vol. 13, p. 210-232. 
Dutt A.K. (1992), “Conflict Inflation, Distribution, Cyclical Accumulation and Crises”, European Journal of Political Economy, vol. 8, p. 579-597.

Eichengreen B. and Hausmann R. (1999), "Exchange rates and financial fragility”, Proceedings of Federal Reserve Bank of Kansas City, p. 329-368.

Fanelli J. M. y Chisari O. (1989), “Restricciones al crecimiento y distribución del ingreso : el caso argentino", Documentos Cedes, $\mathrm{n}^{\circ} 27$.

Frenkel R. (1979), “Decisiones de precio en alta inflación”, Desarrollo Económico, vol. 19, p. 291-331.

Frenkel R. (1980), "El desarrollo reciente del mercado de capitales en la Argentina”, Desarrollo Económico, vol. 20, p. 215-248.

Frenkel R. (1984), “Salarios industriales e inflación. El periodo 1976-1982”, Desarrollo Económico, vol. 24, p. 387-414.

Furtado C. (1958), Perspectivas da economía brasileira, Rio de Janeiro, Ediciones del Instituto Superior de Estudos Brasileiros.

Galbraith J.K. (1957), "Market structure and stabilization policy", Review of Economics and Statistics, vol. 31, p. 124-133.

Graña J.M. y Kennedy D. (2008), "Salario real, costo laboral y productividad”, Documento de trabajo, $\mathrm{n}^{\circ} 12$, IIE-CEPED.

Heredia M. (2006), “La demarcación de la frontera entre economía y política en democracia. Actores y controversias en torno de la política económica de Alfonsín”, in Los años de Alfonsín : ¿El poder de la democracia o la democracia del poder ?, Pucciarrelli, A. (coord.), Siglo XXI, Buenos Aires, p. 153-199.

Heymann D. (1986), “Tres ensayos sobre inflación y políticas de estabilización”, Documento de trabajo de la CEPAL, $\mathrm{n}^{\circ} 18$.

Heymann D. (1987), “The Austral Plan”, American Economic Review, vol. 77, p. 284-287.

Heymann D. (1991), "From sharp disinflation to hyperinflation, twice: the Argentine experience, 1985-1989", in Lessons of Economic Stabilization and Its Aftermath, Bruno M., Helpman E., Liviatan N., Fischer S. (eds.), Cambridge (MA), MIT Press, p. 103-130.

Kaldor N. (1982), The Scourge of Monetarism, Oxford, Oxford University Press.

Lavoie M. (1992), Foundations of Post-Keynesian Economic Analysis, Aldershot, Edward Elgar.

Machinea J.L. and Fanelli J.M. (1988), "Stopping Hyperinflation: The Case of the Austral plan in Argentina, 1985-1987", in Inflation Stabilization, The Experience of Israel, Argentina, Brazil, Bolivia and Mexico, Bruno M., Di Tella G., Dornbusch R. and Fischer S. (eds.), Cambridge (MA), MIT Press, p. 111-152.

Marie J. (2010), “Inflation in Argentina during the second Peronist period (1973-1976): A PostKeynesian Interpretation", Review of Political Economy, vol. 22, p. 281-299.

Marques-Pereira J. (2007), “Crecimiento, conflicto distributivo y soberanía monetaria en Argentina", in Salida de crisis y estrategias alternativas de desarrollo, la experiencia argentina, R. Boyer y Neffa J.C. (coord.), Buenos Aires, Miño y Dávila, p. 177-207.

Martinez R. (2003), Estudios macroeconómicas vol. 3, información estadística macroeconómica parte I, Buenos Aires, CEPAL. 
Miotti L. (1995), « Argentine : de la crise de la régulation à la régulation de la crise », in L'innovation, clef du développement. Trajectoires de pays émergents, Nicolas F., Krieger Mytelka L. (dir.), Paris, Masson, p. 193-246.

Noyola Vázquez J. (1956), “El desarrollo económico y la inflación en Mexico y otros países latinoamericanos", Investigación Económica, vol. 16.

Rapoport M. (2007), Historia económica, política y social de la Argentina (1880-2000), $5^{\text {ta }}$ edición, Buenos Aires, Ediciones Macchi.

Robinson J. (1966), “The economics of hyperinflation”, in Collected Economic Papers, Robinson J., vol. 1, Oxford, Basil Blackwell.

Rowthorn R.E. (1977), “Conflict, Inflation and Money”, Cambridge Journal of Economics, vol. 1, p. 215-229.

Salama P. et Valier J. (1990), L'économie gangrenée, Paris, La Découverte.

Schvarzer J. (1987), La política económica de Martínez De Hoz, Buenos Aires, Hyspamerica-CISEA.

Setterfield M. (2007), "The rise, decline and rise of incomes policies in the US during the post-war era: an institutional-analytical explanation of inflation and the functional distribution of income", Journal of Institutional Economics, vol. 3, p. 127-146.

Sgard J. (2007), « Hyperinflation et reconstruction de la monnaie nationale : une comparaison de l'Argentine et du Brésil (1990-2002) », in La monnaie dévoilée par ses crises, volume 1: Crises monétaires d'hier et d'aujourd'hui, Théret B. (dir.), Paris, Éditions de l'EHESS, p. 461-487.

Smith W. (1991), “Conflicto distributivo y política macroeconómica en Argentina”, Revista Mexicana de sociología, vol. 53, p. 71-106.

Susjan A. and Lah M. (1997), "Inflation in the transition economies: the Post-Keynesian View", Review of Political Economy, vol. 9, p. 381-393.

Taylor L. (2004), Reconstructing Macroeconomics: Structuralists Proposals and Critiques of the Mainstream, Cambridge (MA), Harvard University Press.

Vera L. (2010), “Conflict inflation: an open economy approach”, Journal of Economic Studies, vol. 37, p. 597-615.

Weintraub S. (1978), Capitalism's Inflation and Unemployment Crises, Reading (MA), Addison Wesley.

\section{ANNEXES}

\section{Données macroéconomiques, 1975-1981}

\begin{tabular}{|l|l|l|l|l|l|l|l|}
\hline & 1975 & 1976 & 1977 & 1978 & 1979 & 1980 & 1981 \\
\hline PIB $^{(1)}$ & $-0,4$ & $-0,5$ & 7,8 & $-2,8$ & 6,7 & 0,9 & -7 \\
\hline & & & & & & & \\
\hline Inflation $^{(2)}$ & 335,1 & 347,5 & 160,4 & 169,8 & 139,7 & 87,6 & 131,3 \\
\hline & & & & & & & \\
\hline
\end{tabular}




\begin{tabular}{|c|c|c|c|c|c|c|c|}
\hline Indice des salaires réels ${ }^{(3)}$ & 100 & 66,7 & 64,9 & 62,3 & 75,4 & 82,5 & 73,7 \\
\hline Part des salaires dans le PIB $^{(4)}$ & 48,22 & 30,96 & 29,74 & 32,87 & 35,81 & 41,26 & 38,73 \\
\hline Termes de l'échange ${ }^{(5)}$ & 85,87 & 78,96 & 91,68 & 89,58 & 99,20 & 122,55 & 131,76 \\
\hline Prix des importations & 98,13 & 94,17 & 87,63 & 91,13 & 108,05 & 105,60 & 101,87 \\
\hline Prix des exportations & 84,21 & 74,39 & 80,35 & 81,75 & 107,60 & 129,47 & 134,27 \\
\hline
\end{tabular}

(1) INDEC, taux de croissance

(2) Série IPC, INDEC, de décembre à décembre

(3) D'après Beccaria et Orsatti (1985)

(4) Graña et Kennedy (2008)

(5) D'après Coremberg et al. (2007), base $100=1985$

Données macroéconomiques, 1981-1989

\begin{tabular}{|l|l|l|l|l|l|l|l|l|l|}
\hline & $\mathbf{1 9 8 1}$ & $\mathbf{1 9 8 2}$ & $\mathbf{1 9 8 3}$ & $\mathbf{1 9 8 4}$ & $\mathbf{1 9 8 5}$ & $\mathbf{1 9 8 6}$ & $\mathbf{1 9 8 7}$ & $\mathbf{1 9 8 8}$ & $\mathbf{1 9 8 9}$ \\
\hline & & & & & & & & & \\
\hline PIB $^{(1)}$ & -7 & $-5,8$ & 2,6 & 2,2 & $-4,6$ & 5,8 & 1,8 & -3 & $-7,2$ \\
\hline & & & & & & & & & \\
\hline Inflation (2) $^{(1,3}$ & 209,7 & 433,7 & 688 & 385 & 82 & 175 & 388 & 4924 \\
\hline & & & & & & & & & \\
\hline Indice salaires réels $^{(3)}$ & 87,2 & 77,6 & 102,3 & 124,4 & 100,0 & 102,1 & 96,0 & 91,3 & 82,8 \\
\hline & & & & & & & & & \\
\hline Part des salaires $^{(4)}$ & 38,73 & 29,28 & 33,8 & 39,81 & 39,53 & 41,1 & 39,15 & 33,32 & 28,8 \\
\hline Prix des exportations $^{\prime}$ & 134,27 & 110,88 & 106,9 & 116,0 & 100,0 & 92,2 & 94,3 & 108,5 & 111,5 \\
\hline Prix des importations $^{\prime} 101,87$ & 104,9 & 100,4 & 99,9 & 100,0 & 106,8 & 111,2 & 119,5 & 125,8 \\
\hline & & & & & & & & & \\
\hline
\end{tabular}

(1) INDEC, taux de croissance 
${ }^{(2)}$ Série IPC, INDEC, de décembre à décembre

(3) Données BCRA, reprises de Damill et Frenkel (1990)

(4) Graña et Kennedy (2008)

(5) D'après Coremberg et al. (2007), base $100=1985$

Éléments de la balance des paiements, en millions de dollars courants, 1975-1981

\begin{tabular}{|l|l|l|l|l|l|l|l|}
\hline & 1975 & 1976 & 1977 & 1978 & 1979 & 1980 & 1981 \\
\hline & & & & & & & \\
\hline Solde transactions courantes & -1285 & 649,6 & 1289,9 & 1833,6 & $-550,1$ & -4768 & -4714 \\
\hline & & & & & & & \\
\hline Compte de capital et financier & 488 & 751,4 & 1024,7 & $-33,5$ & 4585,9 & 2413,5 & 1410,1 \\
\hline IDE & 0 & 0 & 145,7 & 274,2 & 264,6 & 787,7 & 927,1 \\
\hline Crédits financiers & 499,6 & 161,8 & 1156,1 & 1482,1 & 3643,7 & 1879,1 & 3947,2 \\
\hline Crédits commerciaux & $-308,5$ & $-494,5$ & $-15,3$ & $-422,4$ & 818 & $-115,1$ & -3385 \\
\hline Gouvernement et BCRA* & 296,9 & 1084,1 & $-261,8$ & -1367 & $-140,4$ & $-142,2$ & $-79,6$ \\
\hline & & & & & & & \\
\hline Erreurs et omissions & -1 & $-192,2$ & $-97,4$ & 32,3 & 201,9 & $-298,9$ & $-208,8$ \\
\hline & $-797,6$ & $\mathbf{1 2 0 8 , 8}$ & 2217,2 & $\mathbf{1 8 3 2 , 4}$ & $\mathbf{4 2 3 7 , 7}$ & -2653 & -3513 \\
\hline
\end{tabular}

Source : CEPAL (1986, p. 342) sur données de la BCRA

Note (traduction de la mention sous notre document de référence) : La ligne " gouvernement et BCRA » inclut les opérations d'endettement liées à des situations de déséquilibres de balance des paiements ou avec la politique des réserves. Ces opérations se réalisent à travers la Banque centrale (BCRA) et sont propres à la Banque ou au gouvernement. L'endettement net de la BCRA comprend les prêts du FMI ou des banques étrangères. Les transactions du gouvernement incluent la souscription et l'amortissement de bons du trésor externes et de certains prêts d'organismes étrangers.

Éléments de balance des paiements, en millions de dollars courants, 1982-1989

\begin{tabular}{|l|l|l|l|l|l|l|l|l|}
\hline & 1982 & 1983 & 1984 & 1985 & 1986 & 1987 & 1988 & 1989 \\
\hline Compte courant & -2.348 & -2.461 & -2.391 & -953 & -2.859 & -4.238 & -1.572 & -1.292 \\
\hline
\end{tabular}




\begin{tabular}{|l|l|l|l|l|l|l|l|l|}
\hline Marchandises & 2287 & 3331 & 3523 & 4582 & 2128 & 540 & 3810 & 5374 \\
\hline Exportations & 7624 & 7836 & 8107 & 8396 & 6852 & 6360 & 9134 & 9573 \\
\hline Importations & -5337 & -4505 & -4584 & -3814 & -4724 & -5820 & -5324 & -4199 \\
\hline Services réels & 43 & -400 & -205 & -231 & -573 & -285 & -255 & -252 \\
\hline Services financiers & -4709 & -5408 & -5712 & -5304 & -4416 & -4485 & -5127 & -6422 \\
\hline Dividendes & -316 & -425 & -439 & -425 & -482 & -558 & -660 & -664 \\
\hline Intérêts payés & -4926 & -5423 & -5537 & -5132 & -4291 & -4145 & -4678 & -6023 \\
\hline Intérêts reçus & 533 & 440 & 264 & 253 & 357 & 218 & 211 & 265 \\
\hline Transferts & 31 & 16 & 3 & & 2 & -8 & & 8 \\
\hline Compte de capital & -3.808 & -111 & 647 & 397 & 784 & 138 & 191 & -5.593 \\
\hline Dont : & & & & & & & & \\
\hline Investissement direct étranger & 257 & 183 & 269 & 919 & 574 & -19 & 1147 & 1028 \\
\hline Prêts et titres gouvernementaux & 267 & 2136 & 2087 & 974 & 127 & -195 & -356 & -686 \\
\hline Prêts FMI & -6.156 & -2.572 & -1.744 & -556 & -2.075 & -4.100 & -1.381 & -6.885 \\
\hline Autres (erreurs et omissions incluses) & -1032 & -456 & 347 & 361 & 887 & -89 & 247 & -4153 \\
\hline Solde & & 1178 & & 1007 & 547 & 1253 & 541 & 233 \\
\hline
\end{tabular}

Source : D'après données BCRA, en millions de dollars

\section{NOTES}

1. La réflexion théorique est similaire avec la dynamique présentée par le modèle néo-kaleckien développé par Charles et Marie (2012). Ce modèle vise à explorer les conditions d'apparition de l'hyperinflation et met l'accent sur le conflit de répartition, l'évolution de l'endettement externe et les anticipations sur le change. Cette référence peut ainsi fournir au lecteur intéressé une trame analytique différente dans sa présentation mais pleinement cohérente avec la réflexion développée ici.

2. À l'exception notable de Carvalho au début des années 1990.

3. L'article, dans une version étendue, figure dans les Collected Economics Papers (Robinson, 1966). L'ouvrage de Besciani-Turroni est quant à lui considéré comme la référence académique orthodoxe concernant l'hyperinflation allemande de 1921. D'abord publié en italien en 1931, l'ouvrage est traduit puis publié en anglais en 1937.

4. La vitesse d'ajustement des comportements est bien sûr un élément qui a son importance. Pour un modèle prenant en compte cet aspect, on peut proposer de se référer à Dalziel (1990). 
5. Les gains de productivité sont susceptibles de diminuer le rythme inflationniste. Si on admet la possibilité d'obtention d'une situation d'état stationnaire, la part des salaires dans la valeur ajoutée (respectivement la part des profits) est alors constante. Cela signifie que le taux de croissance des salaires est strictement égal au taux de croissance des prix. Cette relation, si l'on considère une absence de gains de productivité, peut s'écrire: $\dot{\omega}=w^{n}-p=0$, avec $\dot{\omega}$ la variation de la part des salaires dans la valeur ajoutée, $\dot{w}$ le taux de croissance des salaires et $\dot{p}$ le taux de croissance des prix.

Si on inclut la variation de la productivité (notée $\alpha^{\widetilde{\alpha}}$ ), la relation d'état stationnaire précédente devient : $\dot{\omega}=\dot{w}-\dot{p}-\dot{\alpha}=0$. Les progrès de la productivité $(\dot{\alpha}>0)$ peuvent donc ralentir l'inflation (c'est-à-dire diminuer la valeur de $\dot{p}$ ) par rapport à la situation initiale et au partage de la valeur ajoutée constant. Pour des développements concernant l'importance des variations de la productivité dans le conflit de répartition, on peut se reporter à Lavoie (1992) ou Cassetti (2012).

6. Les principales statistiques macroéconomiques utilisées dans cet article sont regroupées en annexe.

7. Isabel Perón, élue vice-présidente en octobre 1973, est devenue présidente lorsque son mari, Juan Perón est décédé le $1^{\mathrm{er}}$ juillet 1974 . Elle est destituée par une junte militaire qui place à la tête de l'État le général Videla.

8. La récession apparaît une fois la libéralisation financière effectuée. Sur les 6 premiers mois de l'année, l'inflation mensuelle moyenne est de $7.33 \%$ contre $9.31 \%$ sur la fin de l'année. Il y a bien une augmentation du rythme inflationniste moyen, et le pays entre en récession à la suite des réformes étudiées précédemment.

9. Pour une discussion théorique (mais orthodoxe) de la Tablita et de ses liens avec l'approche monétaire de la balance des paiements, on peut se reporter à Blejer et Mathieson (1981). Nous pouvons aussi souligner que cette filiation théorique est revendiquée au sein des documents contemporains de la Banque centrale, comme dans son Memoria Anual de l'année 1979.

10. Avec un taux de dévaluation de 5,2\% en janvier 1979, le différentiel de taux d'intérêt du dollar avec le peso prenant en compte la variation du taux de change est de 0,81 \%. Ce différentiel est constamment positif pendant la période de la Tablita, montrant même une tendance à l'augmentation; d'abord suite à l'augmentation des taux observés aux États-Unis en août 1979, puis au cours de l'année 1980, à mesure que se développent les craintes quant au maintien du régime de change.

11. Fanelli et Chisari (1989), estiment que la dette publique externe est passée de 12496 millions de dollars courants en 1978 à 35671 à la fin de 1981. En 1978, la part de la dette privée dans le total de la dette externe était de $35 \%$ contre $44 \%$ en 1981 , $35 \%$ en 1982 et $30 \%$ en 1983. La dette externe totale est alors de 45097 millions de dollars.

12. Machinea et Fanelli (1988, p. 121-122) relèvent que la durée moyenne de tous les types de contrats se réduit dès le début des années 1980. Dans ce contexte, un flux croissant d'informations concernant l'environnement économique est nécessaire à l'ensemble des agents. En d'autres termes, les négociations contractuelles intègrent de nouveaux éléments afin d'éviter de multiplier les coûts de transaction qui seraient provoqués par de nouvelles négociations. L'ensemble des agents prend en compte les variations immédiates des tarifs publics (électricité par exemple), chaque agent privé ayant le même intérêt à voir les variations de ces prix déclencher des hausses équivalentes de ses revenus nominaux (prix et salaires).

13. L'indice des salaires réels fourni par Damill et Frenkel (1990) qui était de 102,3 en 1983 atteint 124,4 en 1984 (base $100=1985$ ).

14. La confiance joue un rôle primordial dans la réussite et les échecs des réformes monétaires. Marques-Pereira (2007) analyse conjointement l'évolution du conflit de répartition et du régime monétaire en Argentine sur une période plus longue (1950-2005) que celle retenue ici, en ayant notamment recours au cadre théorique basé sur le concept de confiance dans la monnaie 
développé par Aglietta et Orléan (2002). Dans le cas argentin, ces crises de confiance trouvent leurs origines dans l'absence d'un compromis stable sur la répartition, dans la succession des coalitions politiques et dans la répétition de réformes monétaires brutales et importantes.

15. L'introduction d'une échelle de conversion démontre que les autorités estiment que toutes les relations contractuelles (loyers, salaires, factures, opérations financières...) intègrent un taux d'actualisation d'environ $23 \%$ mensuel, qui permet aux contractants d'anticiper l'inflation. L'échelle impose un taux de conversion quotidien. Par exemple, si un agent argentin avait pris un engagement contractuel de 10.000 pesos avant la réforme monétaire et qui se trouvait échu au $1^{\text {er }}$ juillet, l'engagement ne sera pas de 10 Australs mais de 8,73 Australs, la valeur de conversion indiquée par l'échelle étant à cette date de 0,873009.

16. Ce problème n'est pas propre à l'Argentine, et est connu au sein de la littérature sous l'appellation générique « du péché originel » (Eichengreen et Hausmann, 1999).

17. On raisonne avec un taux de change de la monnaie domestique exprimé à l'incertain.

18. On peut conseiller de se reporter à Rapoport (2007) pour obtenir une description détaillée de l'histoire économique et sociale de l'Argentine. L'ouvrage comprend notamment un développement de cet épisode.

\section{RÉSUMÉS}

L'article poursuit un double objectif. Le premier vise à proposer une interprétation de l'hyperinflation compatible avec la théorie post-keynésienne. L'analyse des phénomènes hyperinflationnistes par les auteurs post-keynésiens mais aussi par les auteurs structuralistes permet de nourrir notre réflexion. Nous mettons l'accent sur l'importance de la présence d'une devise dans les comportements domestiques au cours des hautes inflations. D'ailleurs, selon nous, l'hyperinflation est indissociable du rejet complet de la monnaie domestique au profit d'une devise. Ce rejet, soudain dans son expression finale, se construit progressivement. Il est suscité par un violent conflit de répartition qui génère une forte inflation, par des phénomènes d'indexation, par un endettement externe libellé en devises et par des comportements autoréalisateurs sur le change.

Le second objectif réside dans la confrontation de cette proposition théorique aux faits économiques observés en Argentine sur une période qui débute lors des années 1970 et mène à l'hyperinflation de 1989. L'analyse d'économie politique historique permet de repérer la séquence suivante: le conflit de répartition est particulièrement virulent et régulièrement reconfiguré dès les années 1970. Cela provoque l'émergence d'arrangements institutionnels développés par les agents (l'indexation), pendant que le dollar prend de plus en plus d'importance dans l'économie. Ce dernier point est encouragé par l'adoption d'une politique de change originale dans un contexte de complète libéralisation financière à la fin des années 1970 . L'impossibilité dans les années 1980 de stabiliser le taux de change dans un contexte de fort endettement externe rend possible en 1989 la survenue de l'hyperinflation, provoquée in fine par une intense spéculation sur le change et un run sur les réserves.

The purpose of the article is twofold. Firstly, it aims at proposing a Post-Keynesian interpretation of hyperinflation. The theoretical building includes elements suggested by Post-Keynesians as well as by structuralists. The accent is put on the role played by a foreign currency in domestic comportments during high inflation periods. Besides, a definition of hyperinflation is suggested: 
hyperinflation could be seen as a substitution by a foreign currency of a domestic currency. This substitution, violent in its final step, is built gradually. Hyperinflation is caused by a violent distributive conflict which generates a strong inflation, by indexation, by an external indebtedness denominated in a foreign currency and by self-fulfilling prophecies.

Secondly, this theoretical framework is confronted to economic facts observed in Argentina from the 1970's to the 1989 hyper-inflationist spurt. The historical political economy analysis allows the spotting of the following sequence: the distributive conflict is highly violent, reshaped several times from the 1970's onwards. It provokes the development of institutional arrangements (indexation), while the dollar becomes more and more important within the economy. This phenomenon is encouraged by the implementation of an original exchange rate regime at the end of the 1970's, in a context of liberalization of the economy. The impossibility to stabilize the exchange rate during the 1980's in a context of external indebtedness makes possible the arising of hyperinflation. It is finally activated by speculation on the exchange rate level and a run on the foreign reserves.

El artículo se propone dos objetivos. El primero se orienta proponer una interpretación de la hiperinflación compatible con la teoría post-keynesiana. El análisis de los fenómenos hiperinflacionarios para los autores post-keynesianos, pero también para los autores estructuralistas, permite alimentar nuestra reflexión. Nosotros ponemos el acento sobre la importancia de la presencia de una divisa en los comportamientos domésticos durante el transcurso de altas inflaciones. Por otra parte, en nuestra opinión, la hiperinflación es indisociable del rechazo completo de la moneda doméstica en beneficio de una divisa. Este rechazo, que a veces llega a su expresión final, se construye progresivamente. Está suscitado por un violento conflicto de distribución del ingreso que genera una fuerte inflación, a través de fenómenos de indexación, por un endeudamiento externo emitido en divisas y por comportamientos autorealizadores sobre el tipo de cambio. El segundo objetivo reside en la confrontación de esa proposición teórica con los hechos económicos observados en Argentina sobre un período que se inicia en los años 70 e incluso en la hiperinflación de 1989. El análisis de economía política histórica permite identificar la secuencia siguiente : el conflicto distributivo es particularmente virulento y regularmente reconfigurado desde los años 70 . Esto provoca la emergencia de arreglos institucionales desarrollados por los agentes (la indexación) mientras que el dólar toma cada vez mas importancia en la economía. Este último punto ha sido estimulado por la adopción de una política de tipo de cambio original en un contexto de completa liberalización financiera al final de los años 70. La imposibilidad durante los años 1980 de estabilizar la tasa de cambio en un contexto de fuerte endeudamiento externo hizo posible que en 1989 sobreviniera la hiperinflación, provocada finalmente por una intensa especulación sobre el tipo de cambio y una corrida sobre las reservas.

\section{INDEX}

Palabras claves : hierinflación, teoría post-keynesiansa, Argentina

Index géographique : Argentine

Keywords : hyperinflation, post-keynesian theory, Argentina

Mots-clés : hyperinflation, théorie post-keynésienne

Code JEL B50 - General, E31 - Price Level; Inflation; Deflation, N16 - Latin America; Caribbean 
AUTEUR

\section{JONATHAN MARIE}

Maître de conférences, université Paris 13, Sorbonne Paris Cité, CEPN (CNRS, UMR 7234),

jonathan.marie@univ-paris13.fr 